\title{
Fermilab
}

Managed by Fermi Research Alliance, LLC for the U.S. Department of Energy Office of Science

\section{Electrospun nanofiber materials for high power target applications}

\section{Sujit Bidhar}

21-22 ${ }^{\text {nd }}$ Sept., 2017

J-PARC, Tokai, Japan

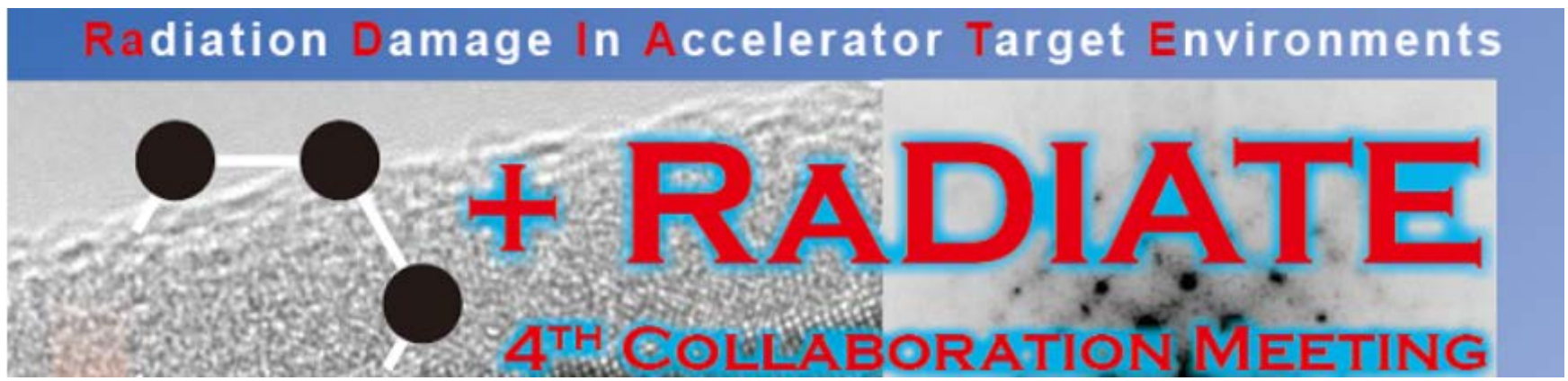

This manuscript has been authored by Fermi Research Alliance, LLC under Contract No. DE-AC02-07CH11359 with the U.S. Department of Energy, Office of Science, Office of High Energy Physics. 


\section{Outline}

- Background \& Objectives

- Electrospinning process

- In-house electrospinning unit

- Candidate target materials manufacturing

- Single nanofiber mechanical characterization

- Future plans and summary 


\section{Background- Target material}

- Demand of multi-MW high performance particle production targets

- LBNE 2.3MW proton beam, 1.6X1014 p/pulse

- Structural integrity over time

- High temperature, thermal stresses, dynamic stresses

- Withstand radiation damage

- Embrittlement, radiation corrosion, swelling 


\section{Current Target}

- ANU/NOvA, 700KW $\rightarrow$ Graphite blocks

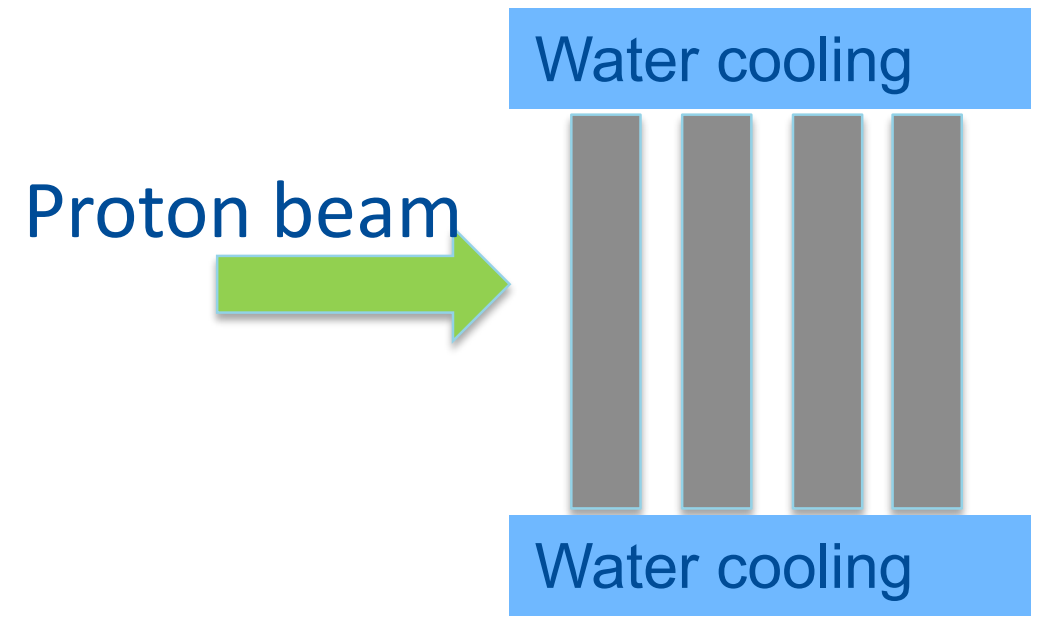

Compressive strength $345 \mathrm{MPa}$ Tensile strength : 60 140 MPa Endurance Limit : $20 \mathrm{MPa}$

Beam spot size $<<$ target dimension Solid continuum $\rightarrow$ high local temperature gradient, thermal stress wave

Can it perform satisfactory at higher energy?? 


\section{HPT Issues -Stress wave}

T2K window

$750 \mathrm{~kW}$

Spill width: 4.2

usec

Gaussian beam

Sigma $4.2 \mathrm{~mm}$
Radial temperature distribution at end of pulse

$476.82 \mathrm{Max}$

457.19

437.56

417.93

398.3

378.67

359.04

339.41

319.78

300.15 Min

Radial stresses

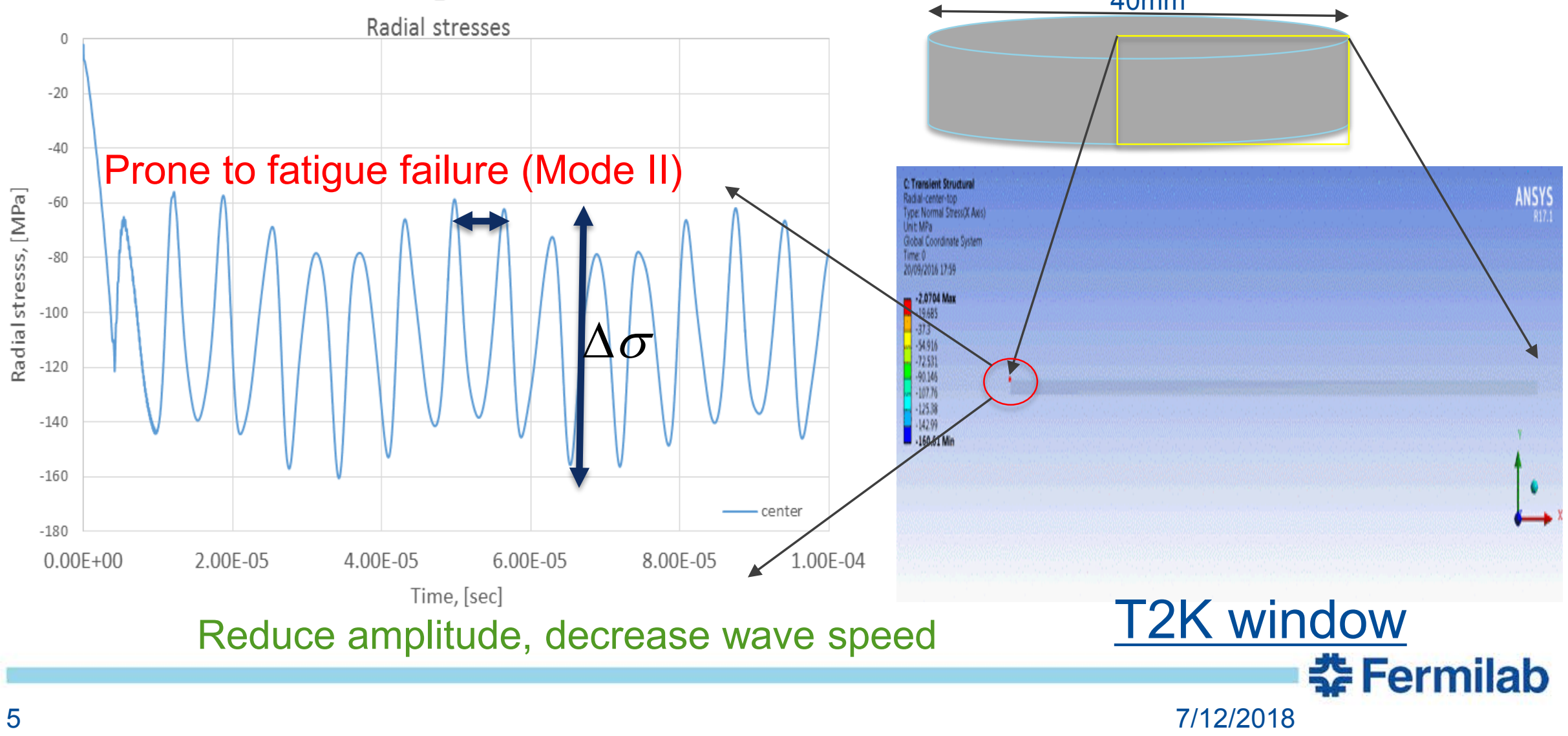




\section{Fatigue life}

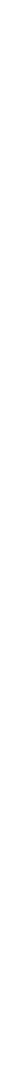

Possible by microstructure design 


\section{Sinuous Target -Candidate Microstructure}

Design Microstructure to mitigate issues

Electrospun nano-fiber $\rightarrow$ Sinuous target
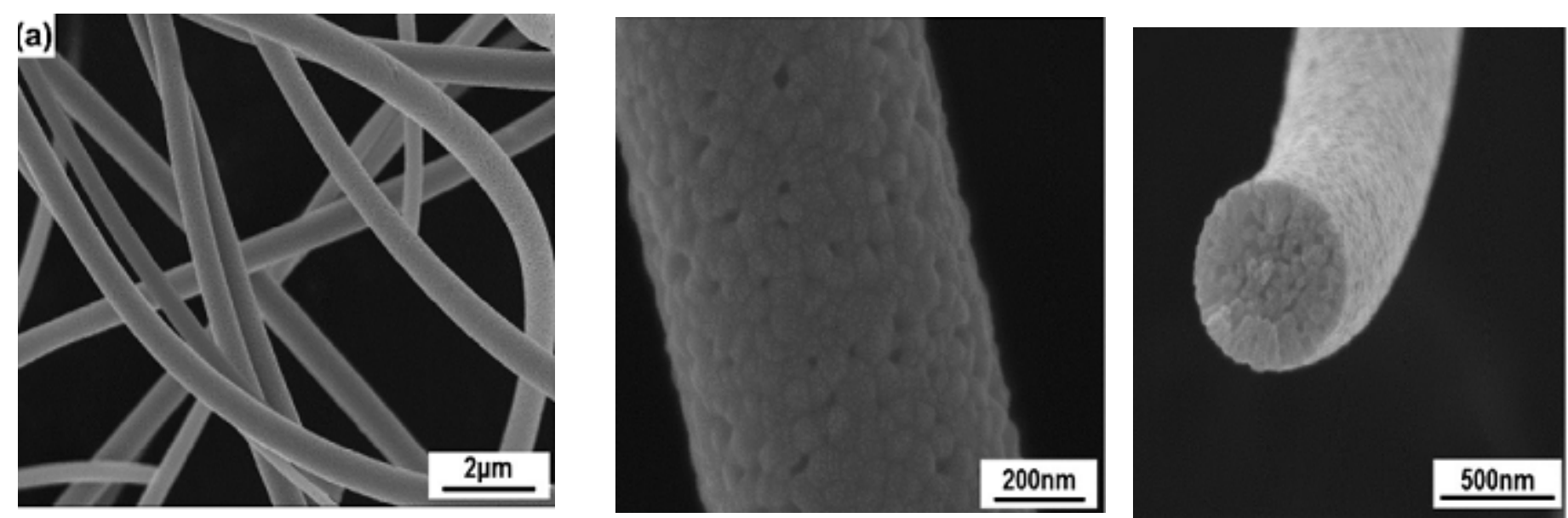

Electrospun Zirconia nanofiber* 


\section{Advantages \& Limitations}

Advantages:

- Local damage of single fiber won't affect target structural integrity as a whole

- Reduced thermal stress wave

- Reduced temp. gradient

- High surface area \& gaps $\rightarrow$ effective local heat removal by passing He gas

- Customize material properties by mixing different materials

Limitations:

- Widely used for polymer nanofibers

- Limited research in ceramics/metal nanofiber

- Low density (long target) 


\section{Objective}

Fabricate ceramic/metal, composite material nano-fiber with high strength, high density and alloying elements to reduce radiation damage.

- Set up in-house electrospun unit

- Fabricate ceramic composites nanofiber

- Micro-mechanic study single fiber mechanical characterization 


\section{Basic Electrospinning Set up}

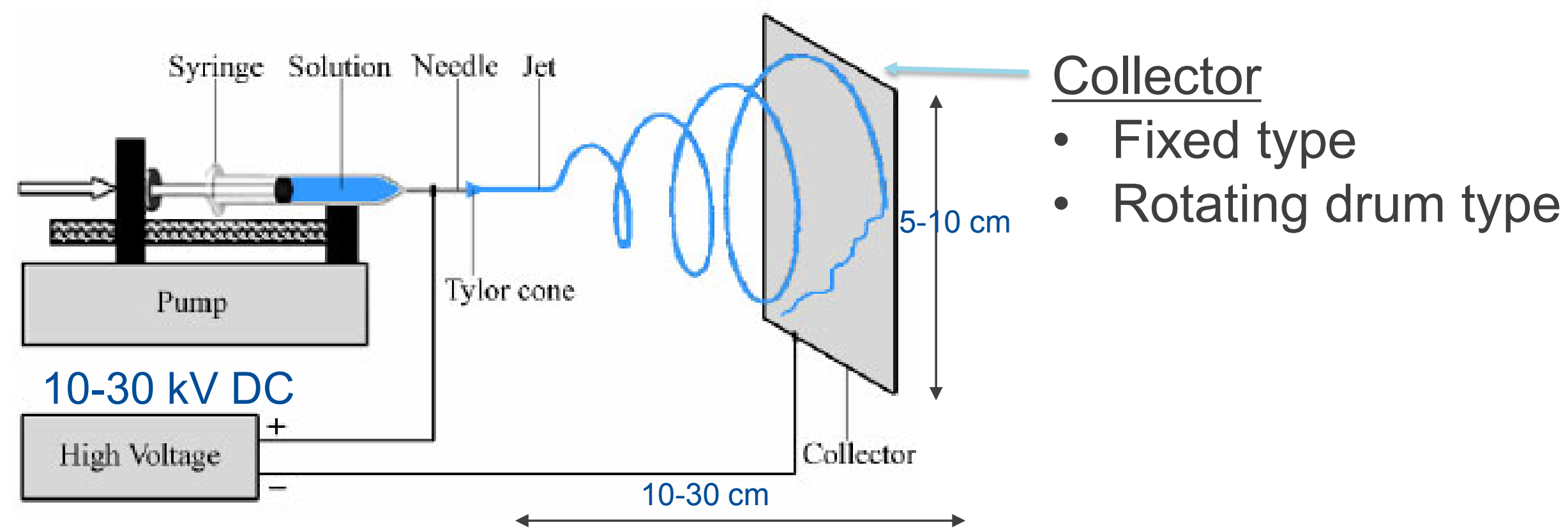

Process carried out at room temp. and atm. pressure 


\section{Electrospinning Principle}

\section{Jet Initiation stages}

\section{을}

Electrostatic repulsion> surface tension

- Droplet is stretched

- Jet elongated by whipping action

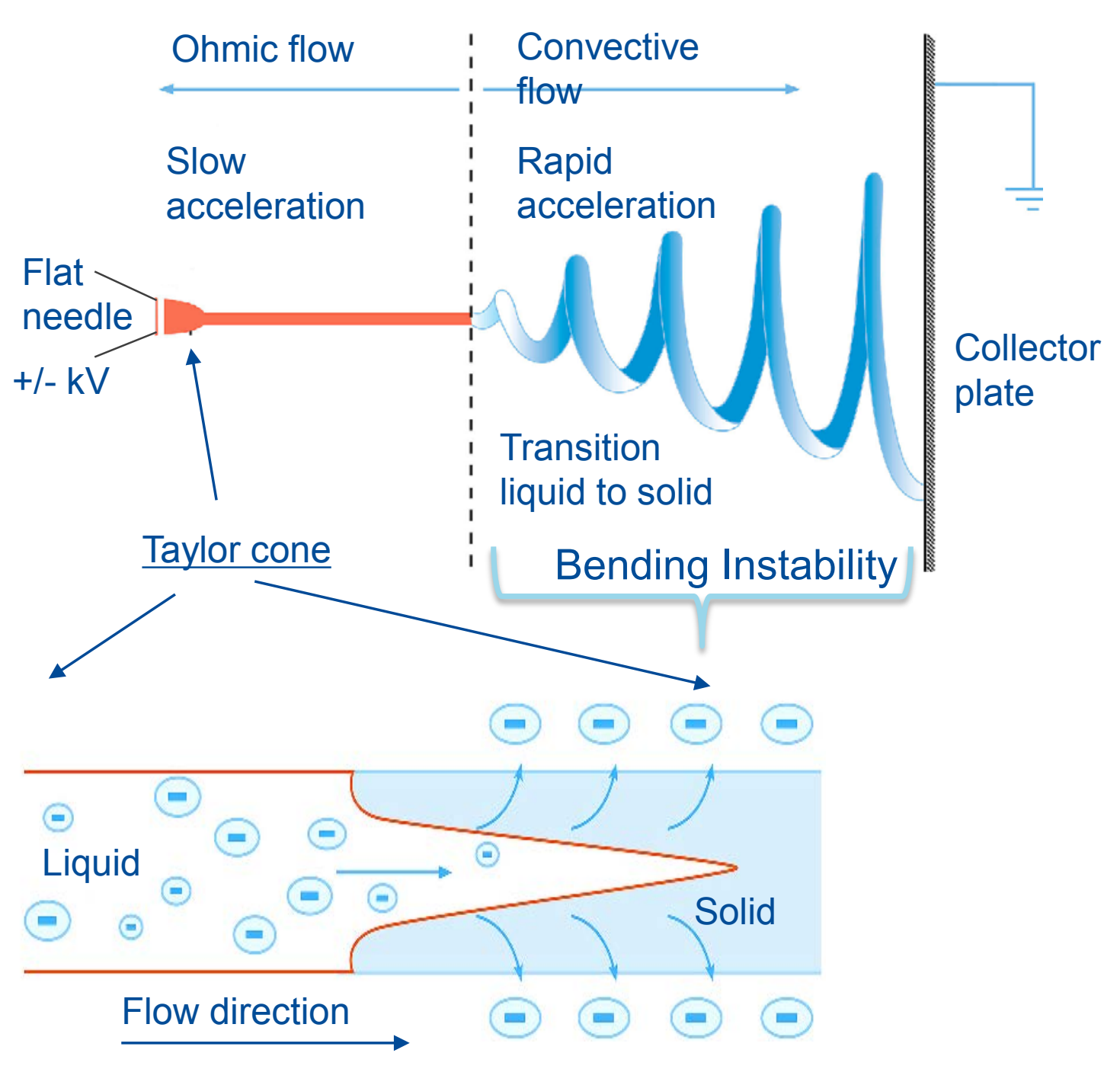

궁 Fermilab 


\section{Ceramics Nanofiber Fabrication}

Inorganic precursor(inorganic compound+solvent)

Polymer solution(polymer+solvent)

\section{Salt additives (surfactants)}

Solution for electrospinning $\rightarrow$ polymer-ceramic nanofiber

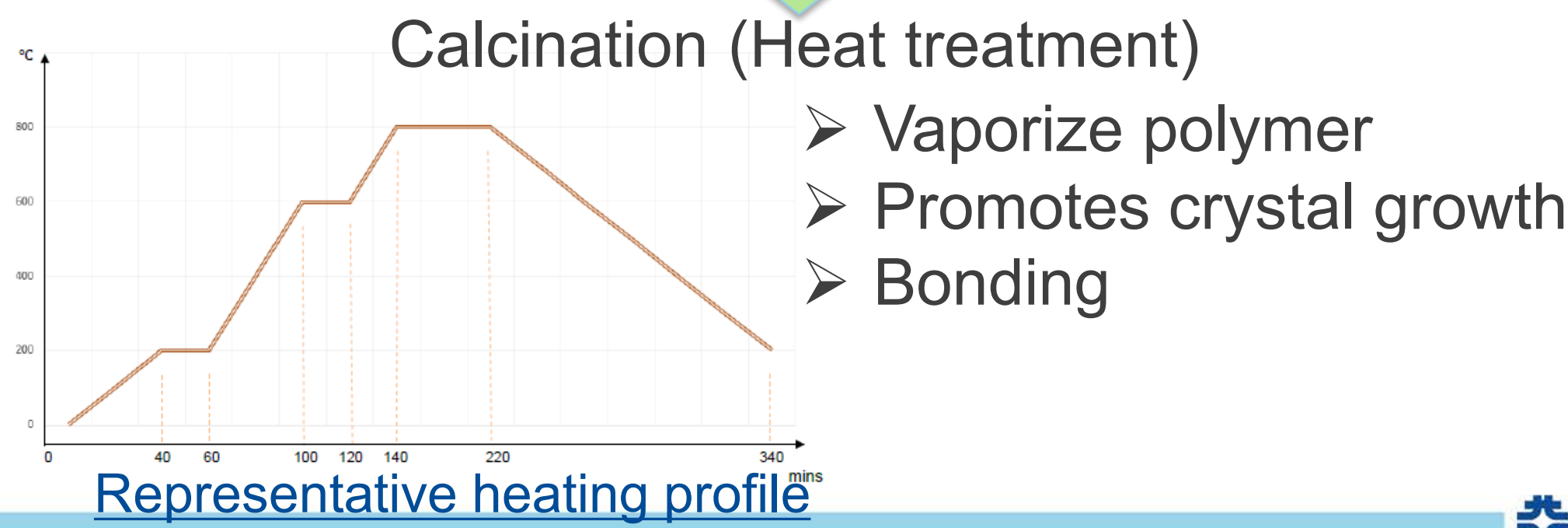




\section{Lab Set-up}

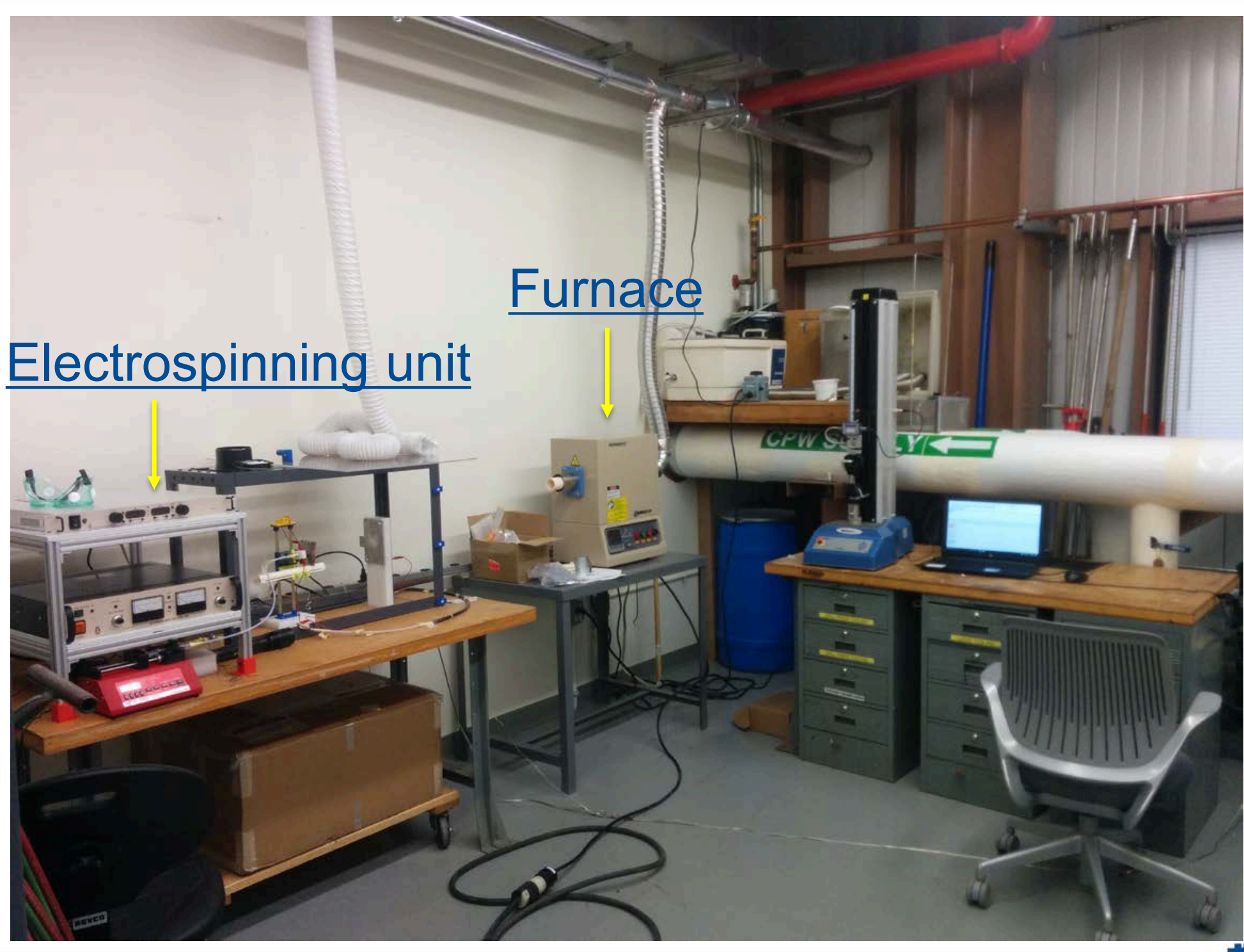

\section{萧 Fermilab}




\section{Electrospinning Set-up}

High voltage power supply

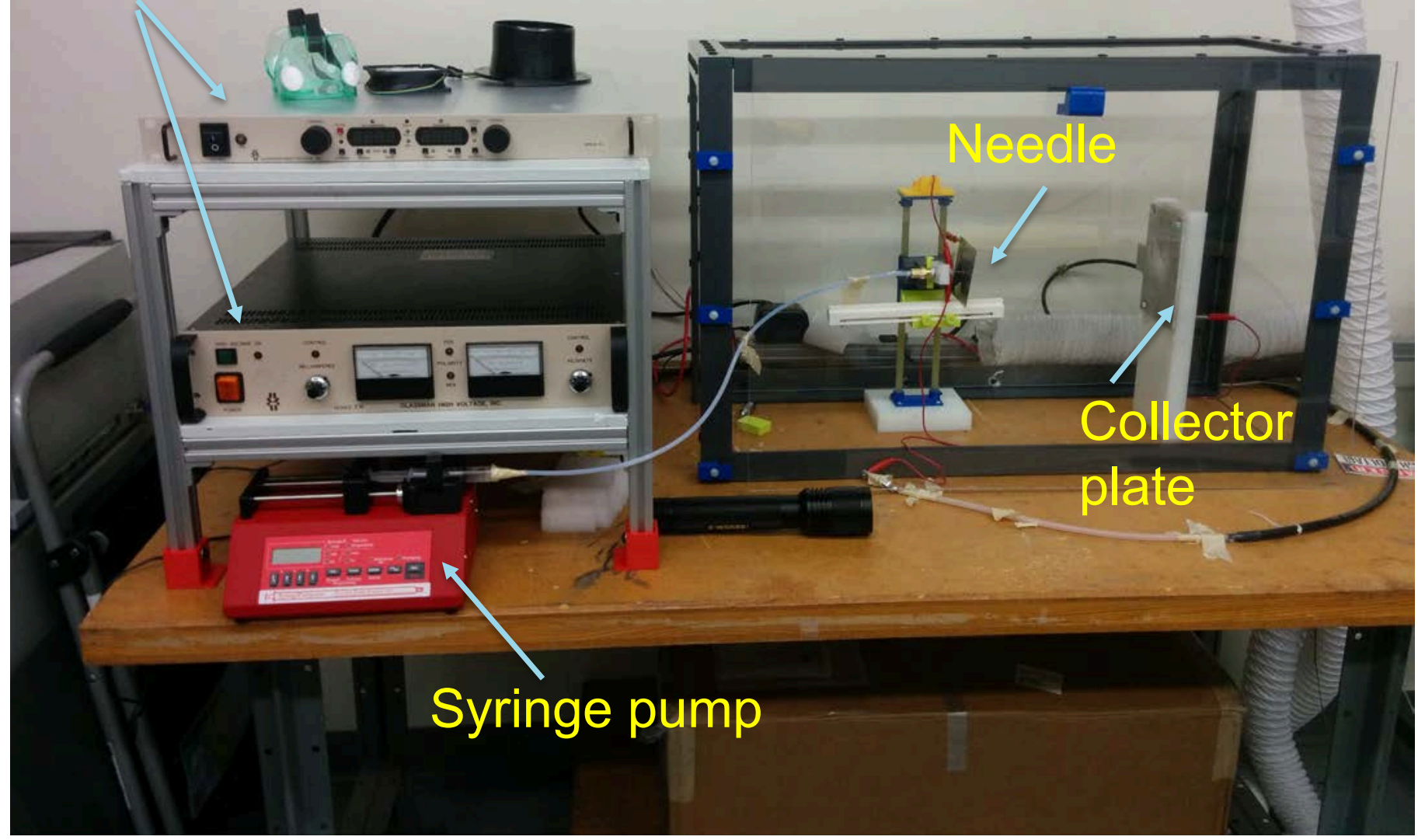




\section{Electrospinning Jet}

Needle

Stable jet 


\section{Nanofiber Mat - Random Orientation}

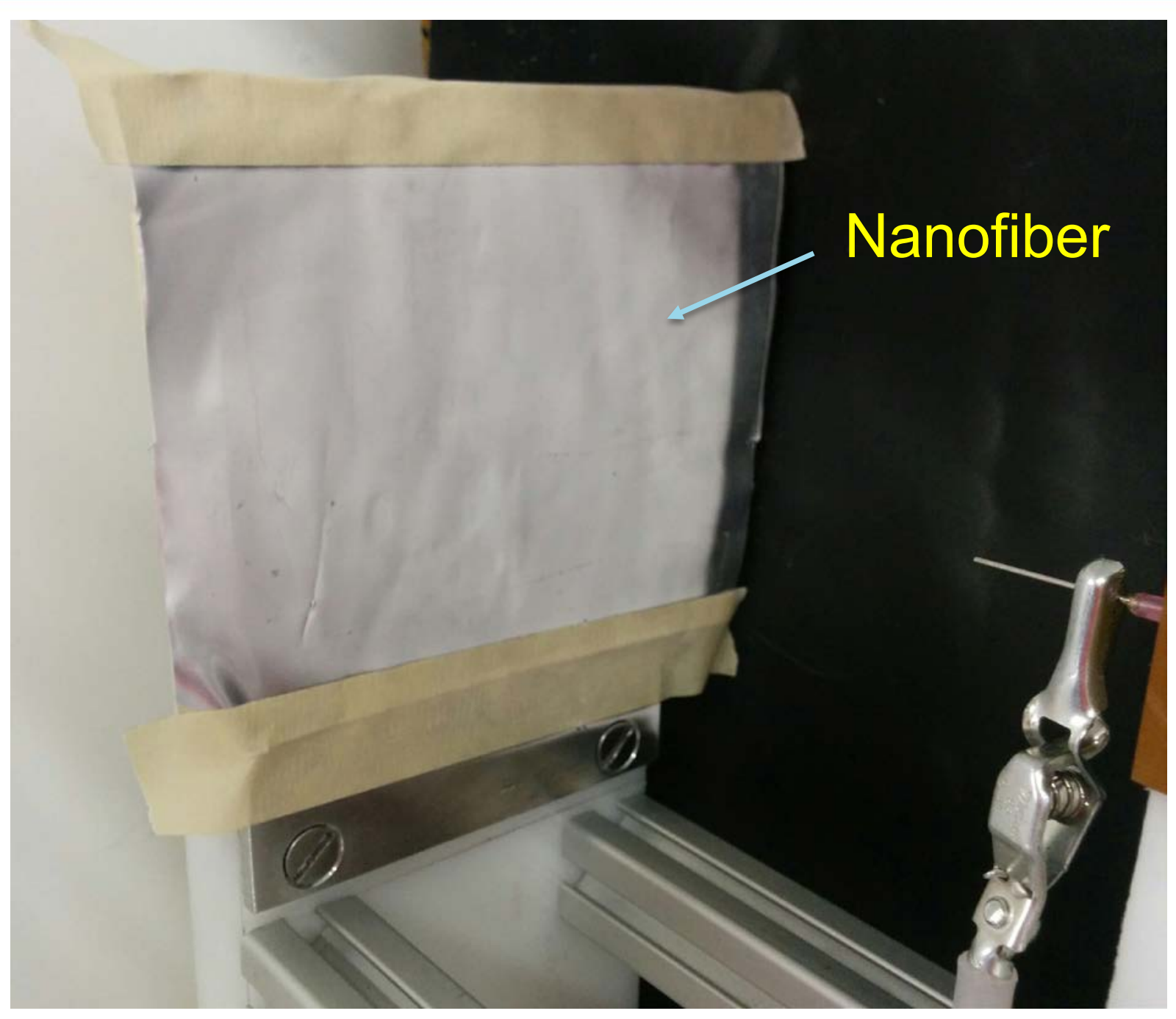




\section{Nanofiber Mat- Aligned}

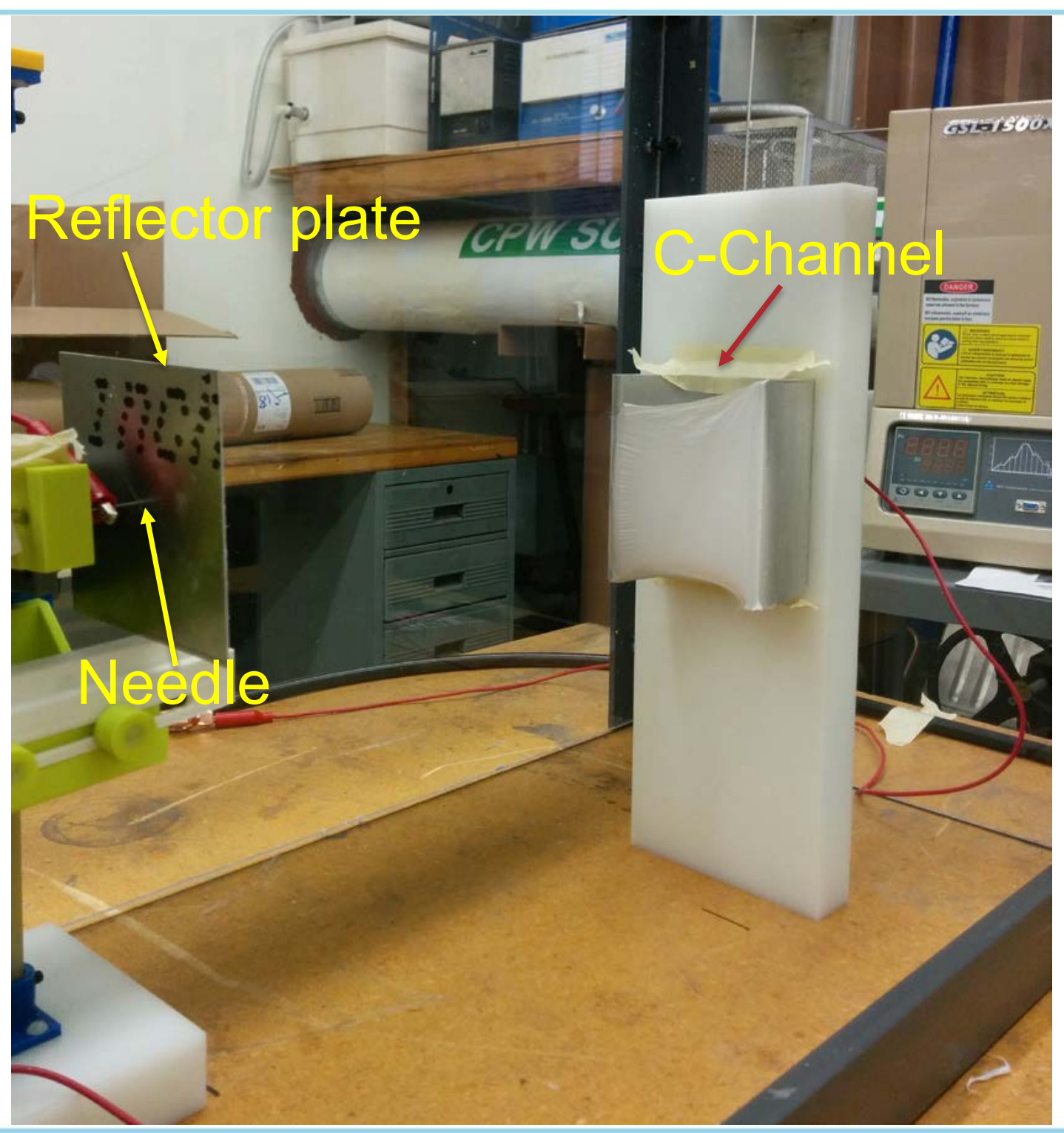

\section{芓 Fermilab}




\section{Focused Deposition}

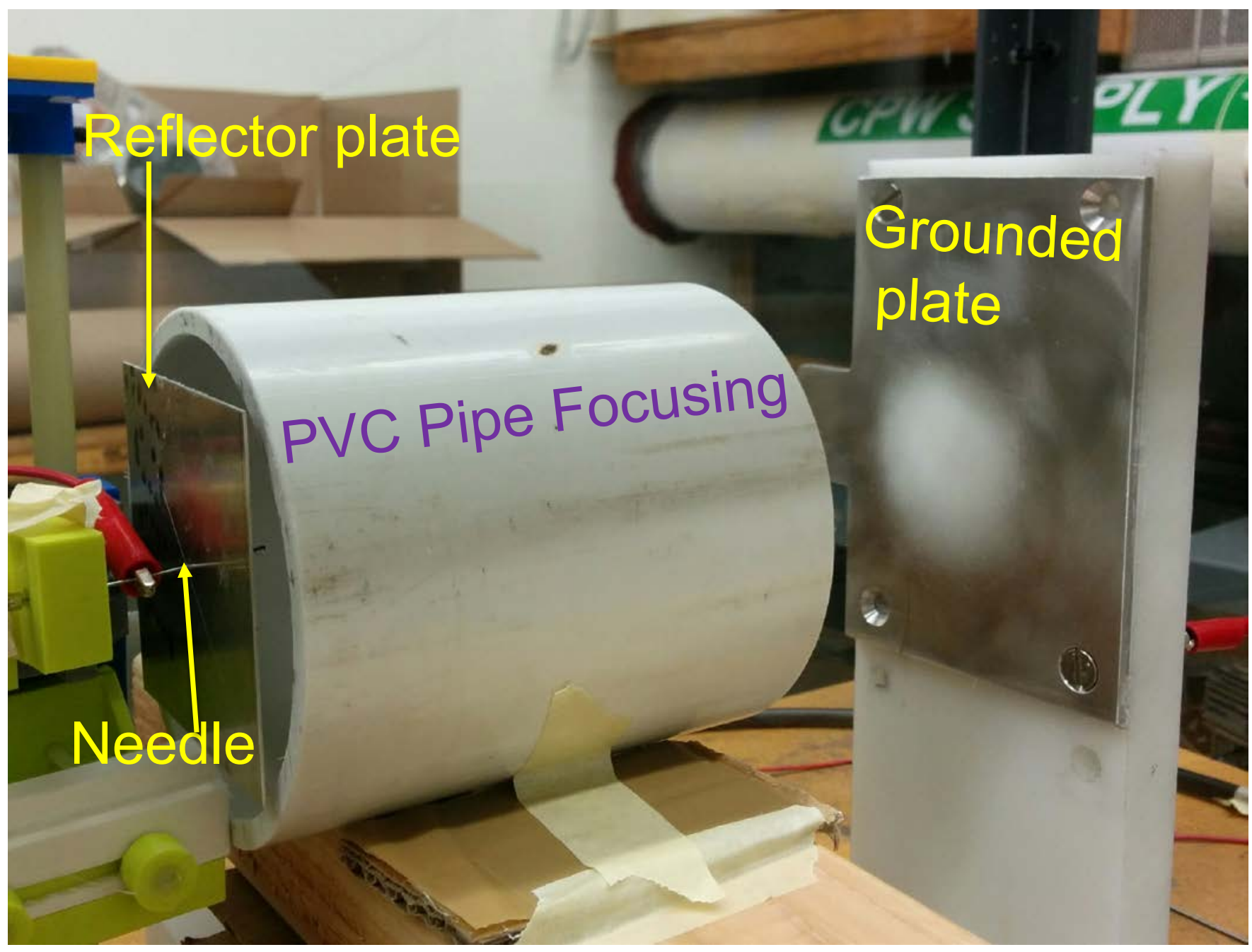

\section{葉 Fermilab}




\section{Improvised Compact Power Supply}

120 Watt, 120VAC in
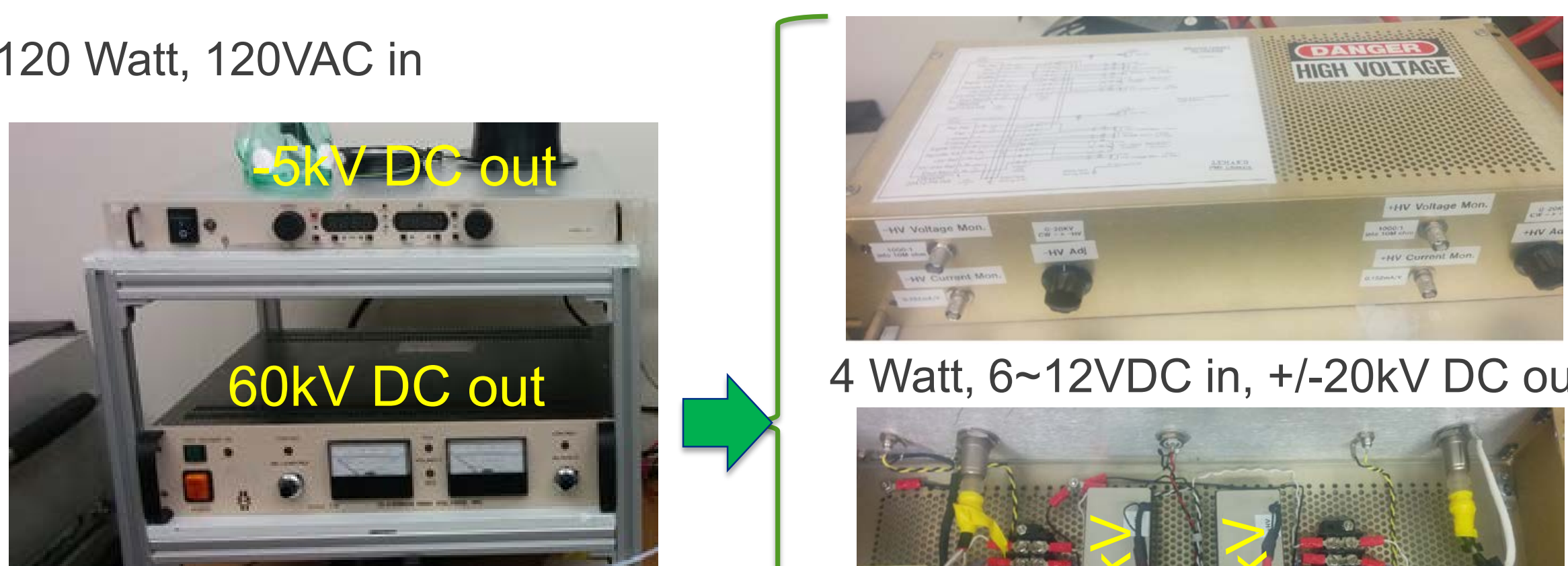

$>$ Much safe to use $(120 \mathrm{~W} \rightarrow 4 \mathrm{~W}$ !)

$>$ Mobile compact unit

- Can be run on 9 or $12 \mathrm{~V}$ battery

4 Watt, 6 12VDC in, +/-20kV DC out

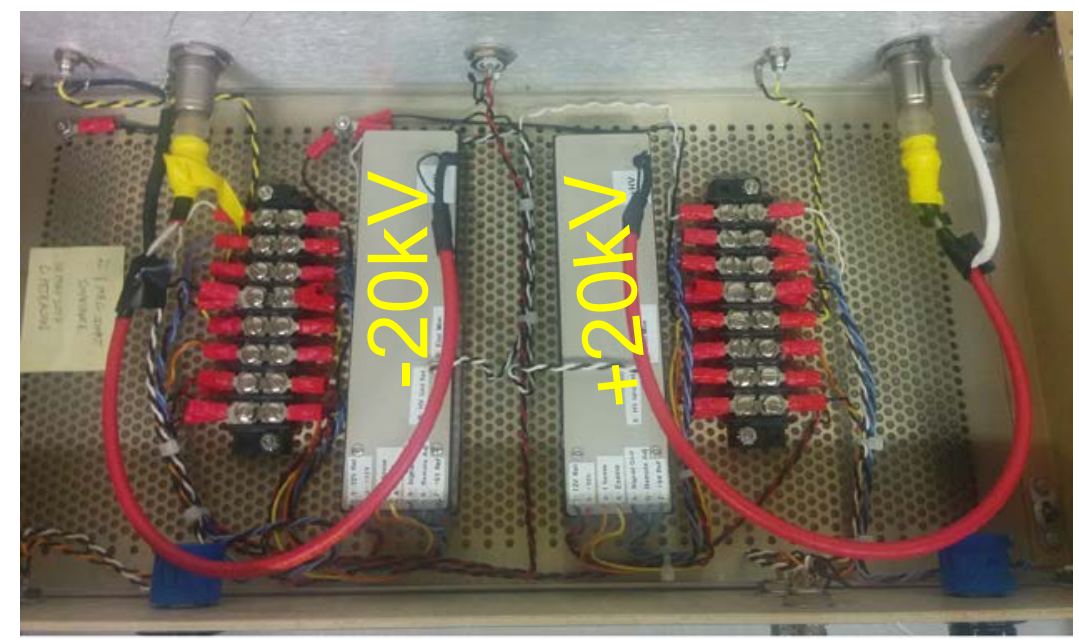




\section{Candidate electrospun nanofiber-Raw materials}

Polymer solution

- PVP+Ethanol+Aceton

Metal/Ceramic

- Alumina $\rightarrow$ Aluminum 2,4-pentadionate+Aceton

- Zirconia $\rightarrow$ Zirconium Carbonate +Acetic Acid

- $\mathrm{WO}_{3} \rightarrow$ Ammonium meta-tungstate + D.I. Water

- $\mathrm{TiO}_{2} \rightarrow$ Titanium Isopropoxide

Carbon-nanotube Composite

- CNT-Alumina

- CNT-Zirconium

Proposed 


\section{Alumina Nanofiber}

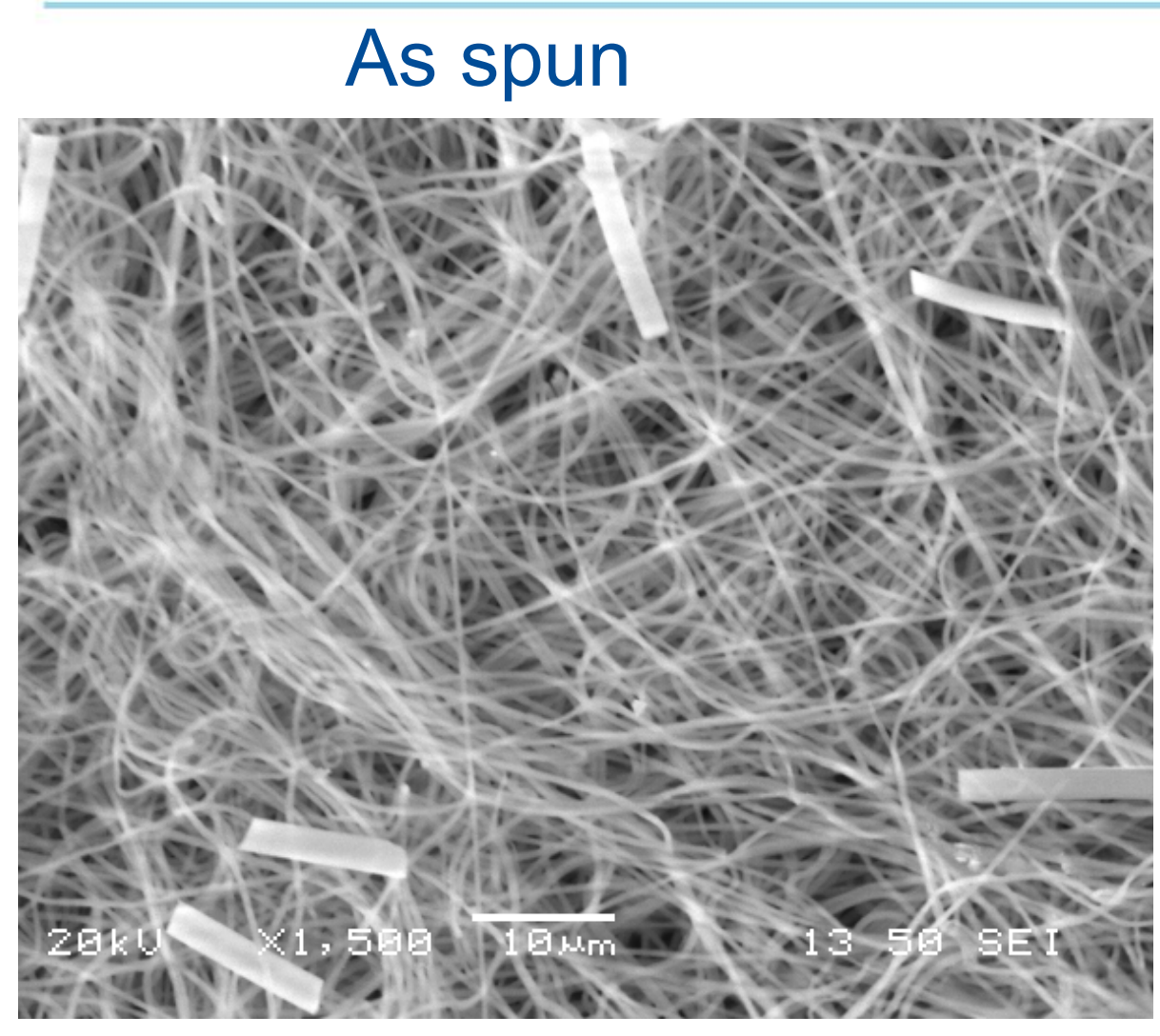

After heat treatment

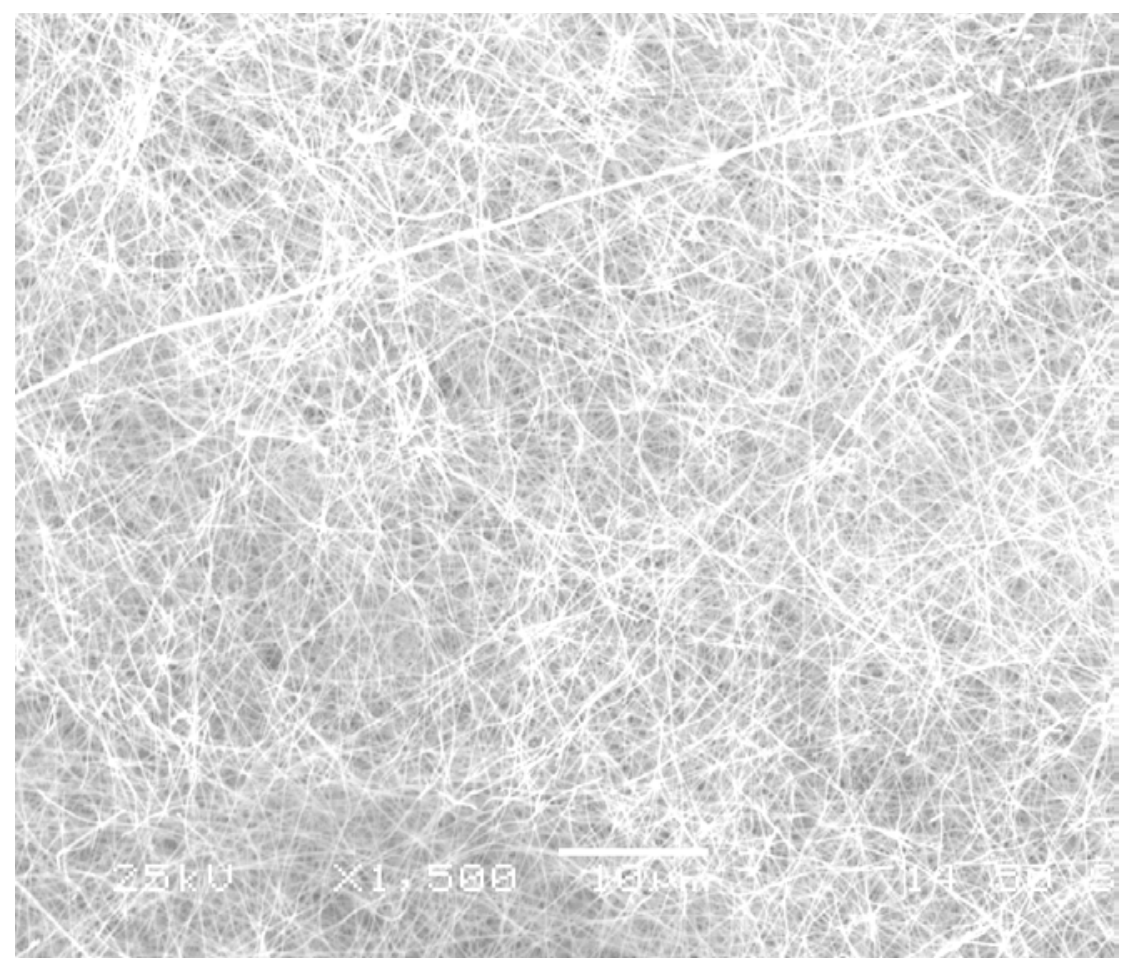




\section{EDS Mapping Alumina Nanofiber}
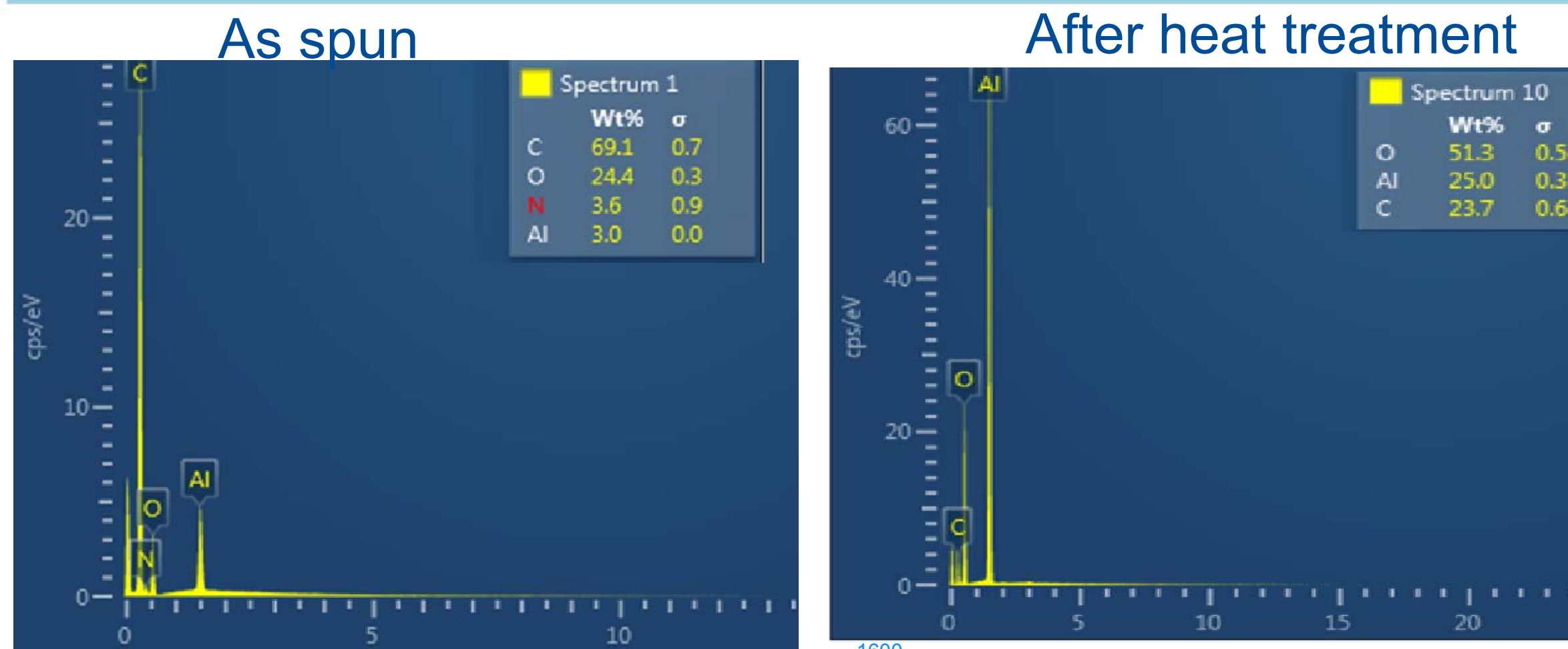

Theoretical $\mathrm{Al}$ wt $\%$ in $\mathrm{Al}_{2} \mathrm{O}_{3}$ is $53 \%$ Achieved in actual $25 \%$

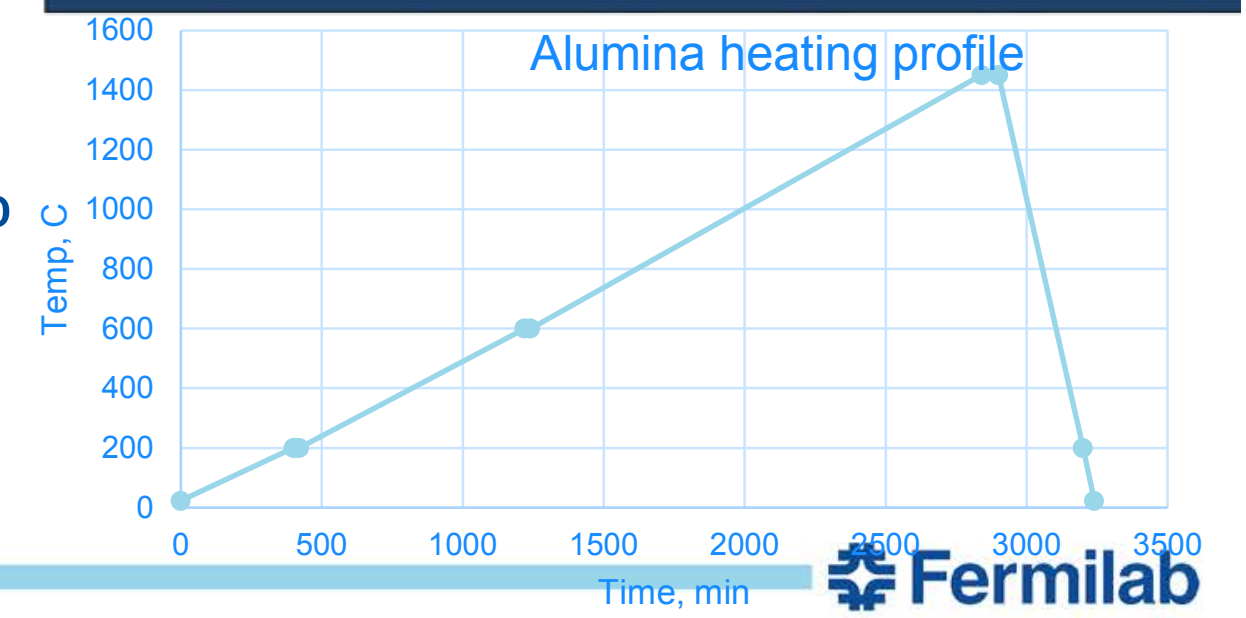




\section{Titania(TiO2) Nanofiber}

\section{As spun}

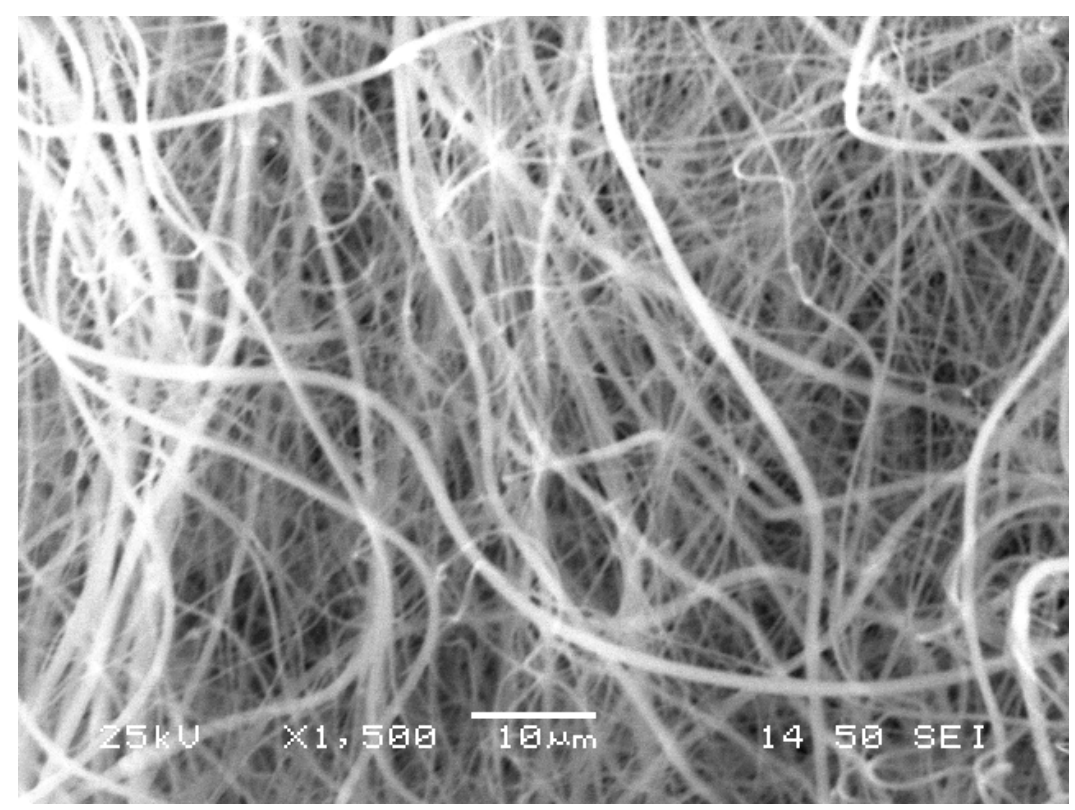

\section{After heat treatment}

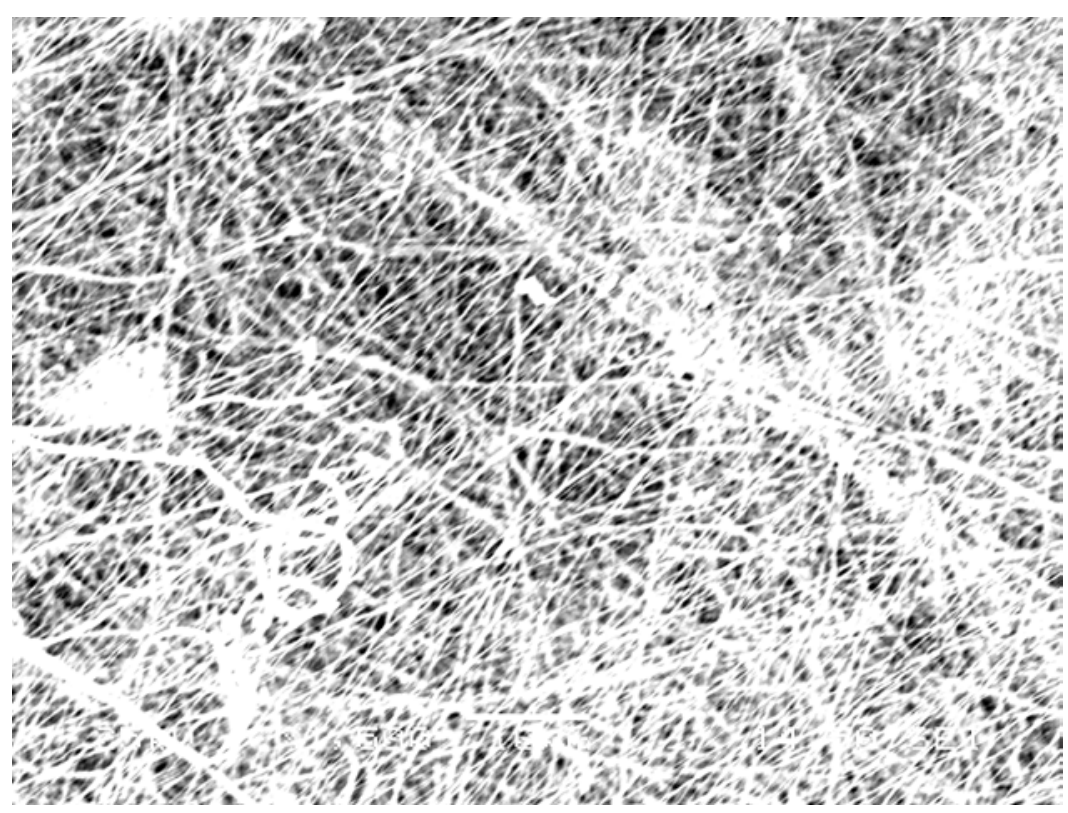




\section{EDS Mapping TiO2}

As spun

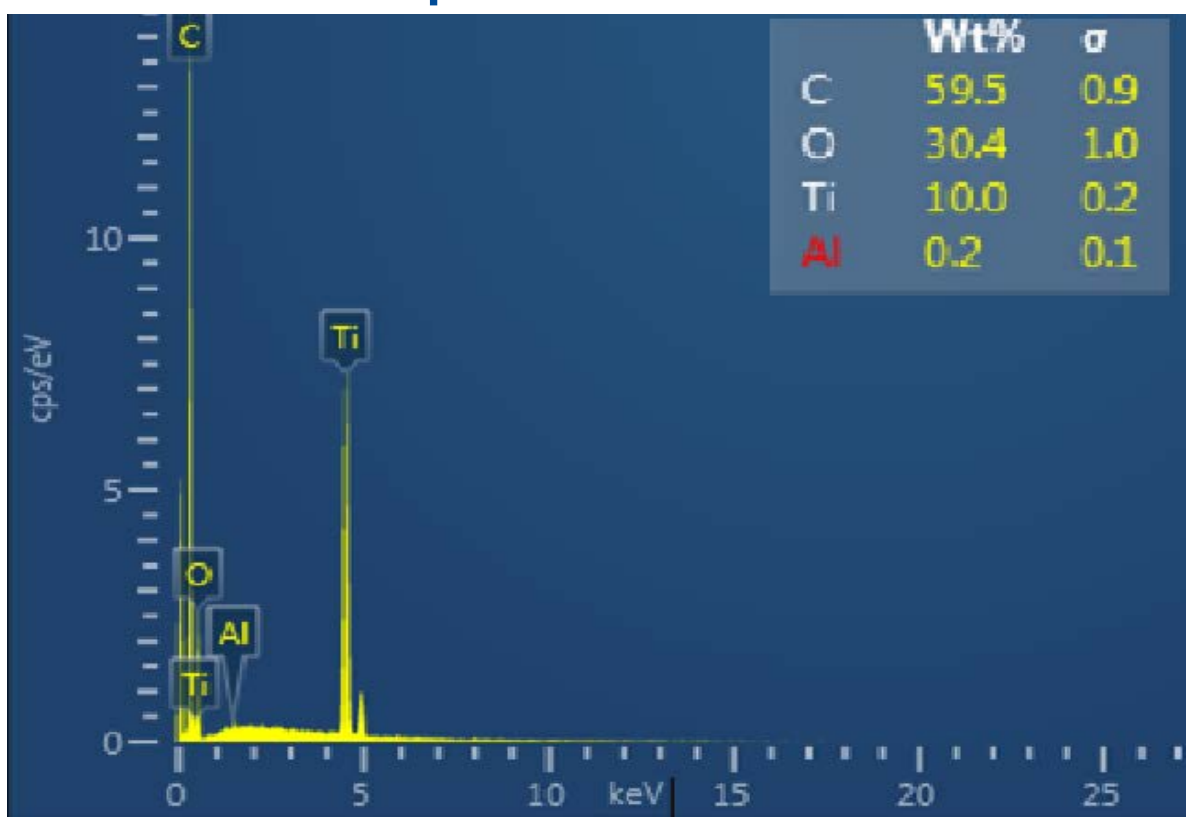

After heat treatment

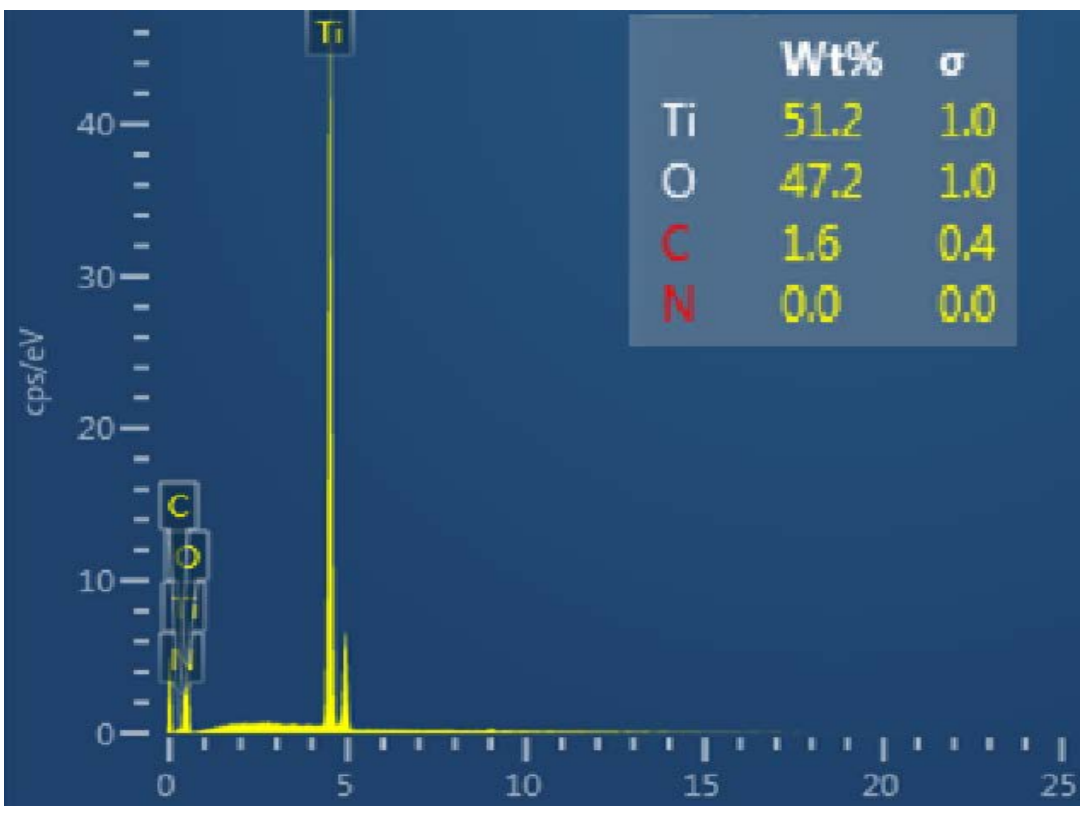

Titanium Heating profile

Theoretical Ti wt $\%$ in TiO2 is $60 \%$ Achieved in actual $51 \%$

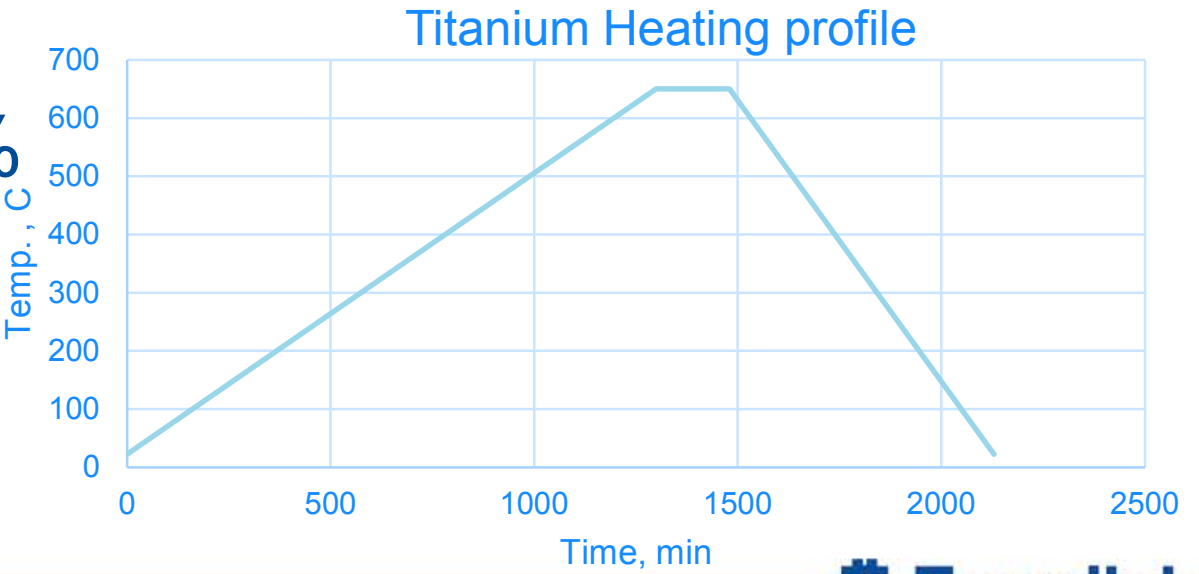




\section{Zirconia Nanofiber}

\section{As spun}

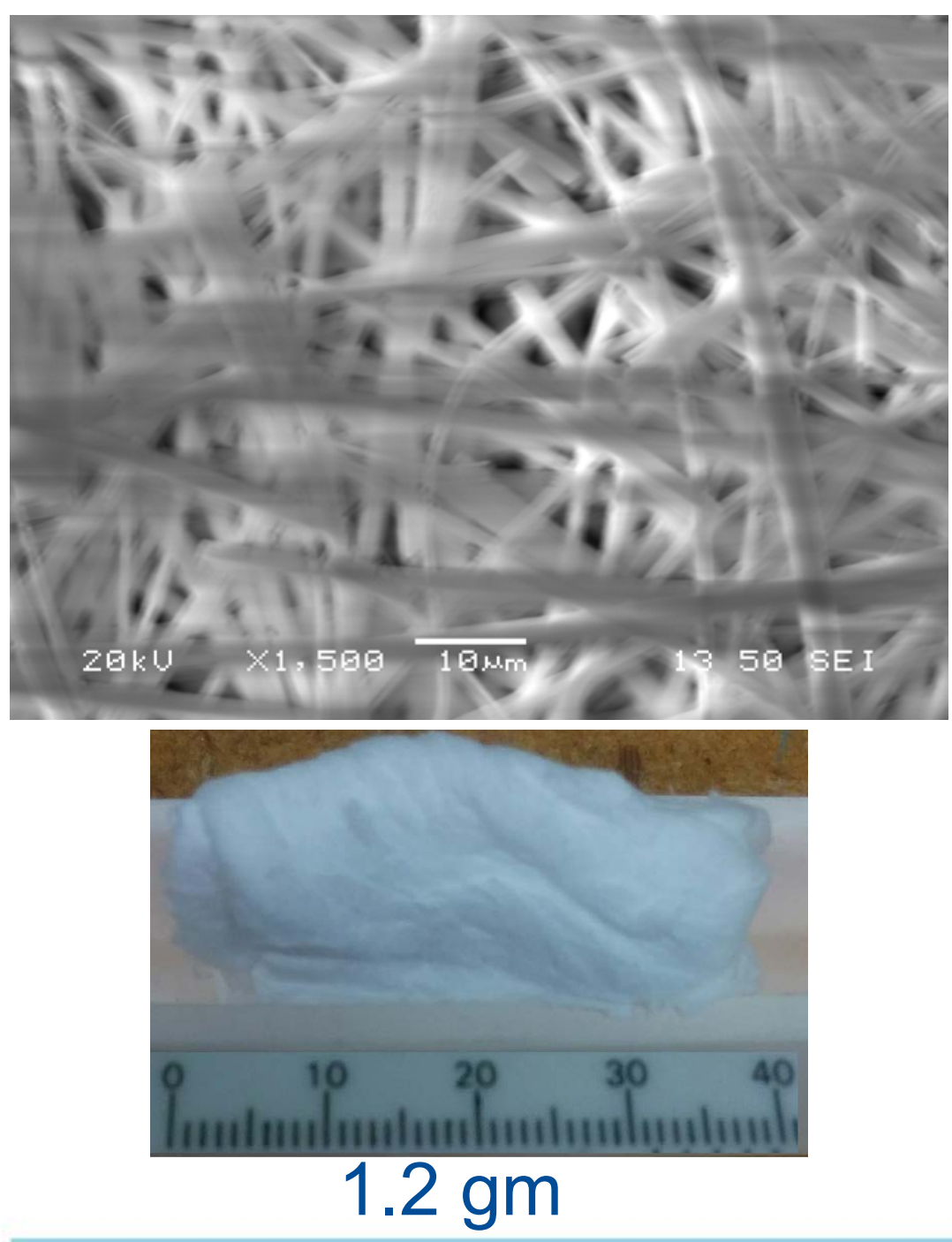

After heat treatment

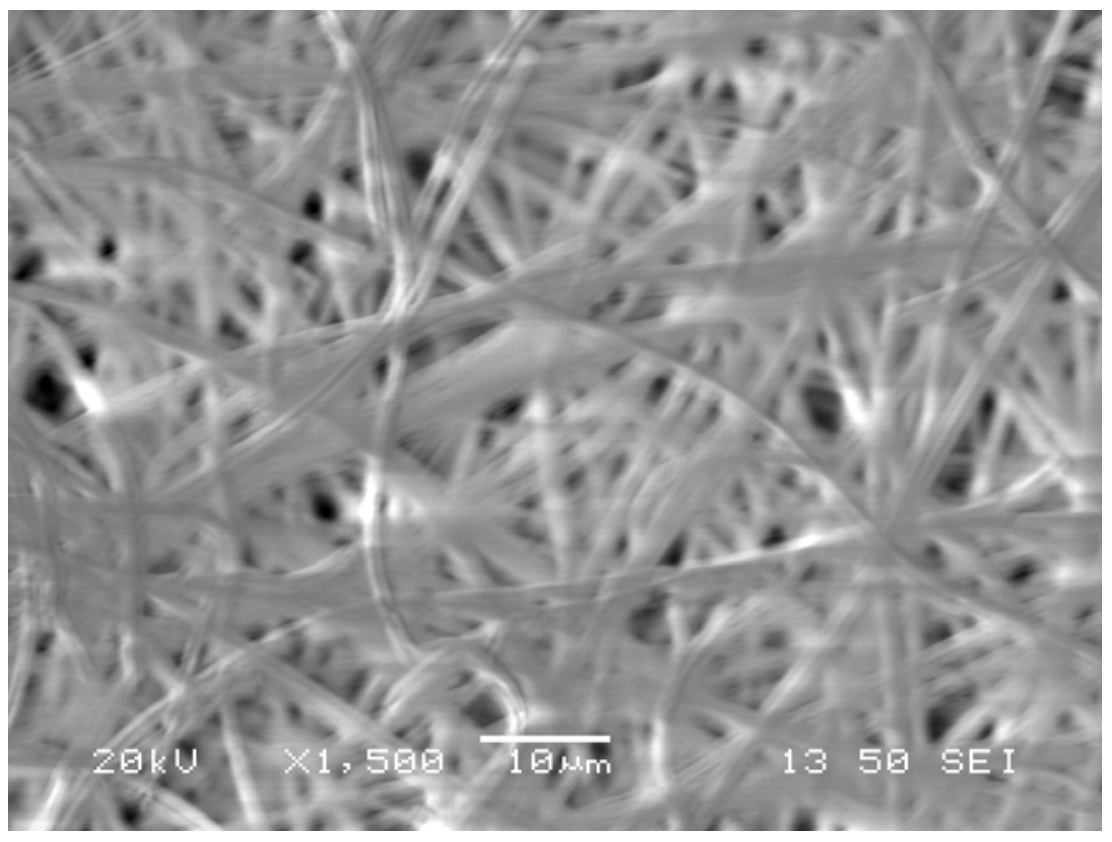

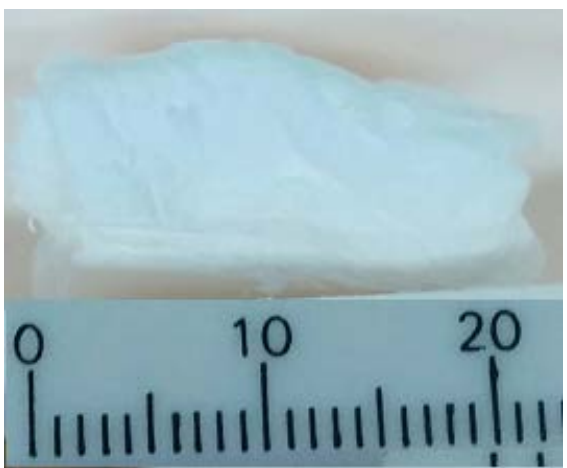
$0.5 \mathrm{gm}$ 


\section{EDS Mapping- Zirconia Nanofiber}

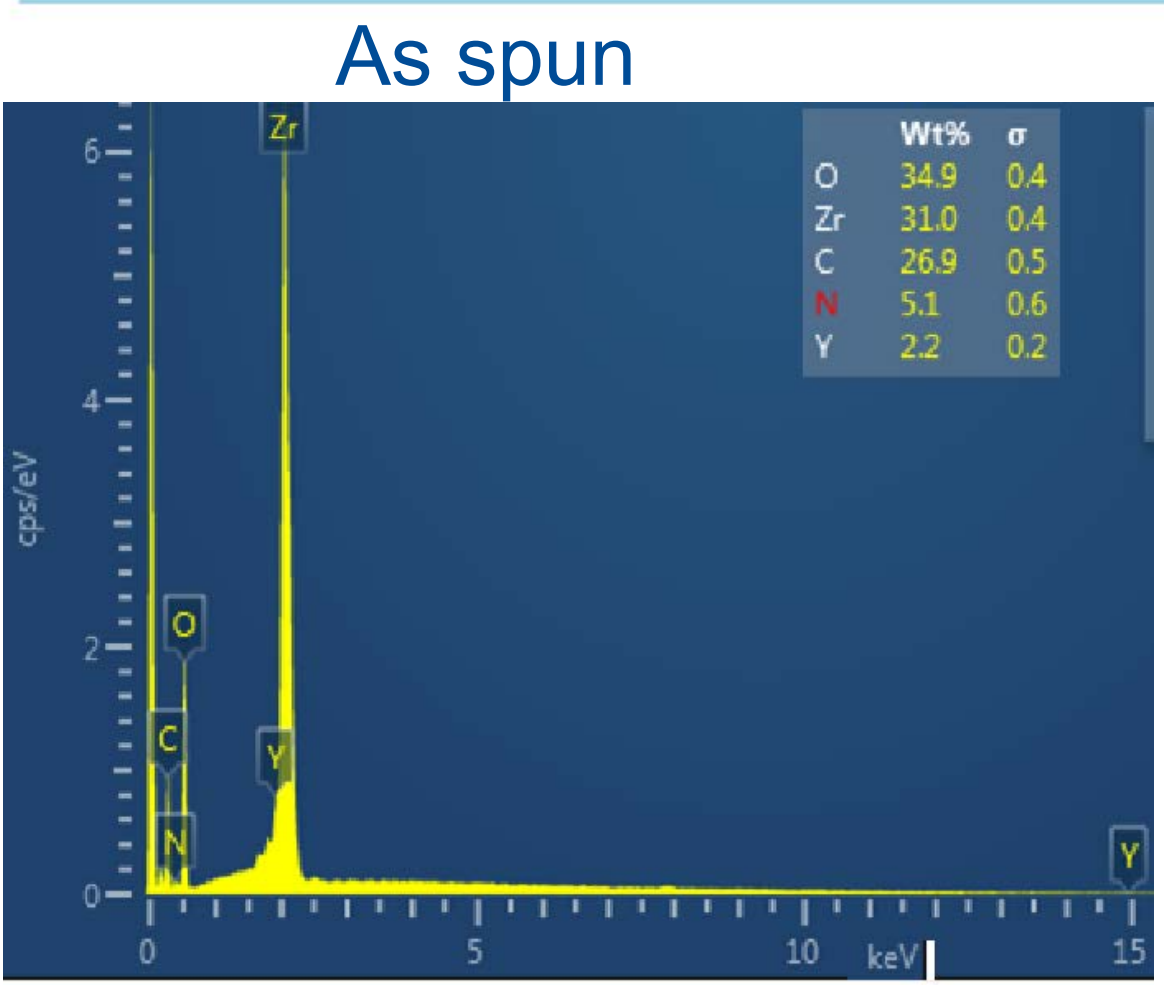

After heat treatment

Theoretical $\mathrm{Zr}$ wt $\%$ in $\mathrm{ZrO}_{2}$ is $74 \%$ Achieved in actual $62 \%$

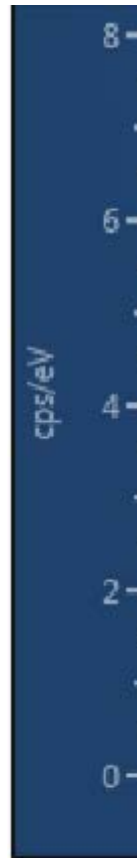

$8-$

$-$

-

$=$

5-

$-$

$-$

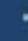

$4-$

$-$

-
-
$2-$
-
-
-
$0-$
0
1400
1

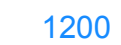

ํ.

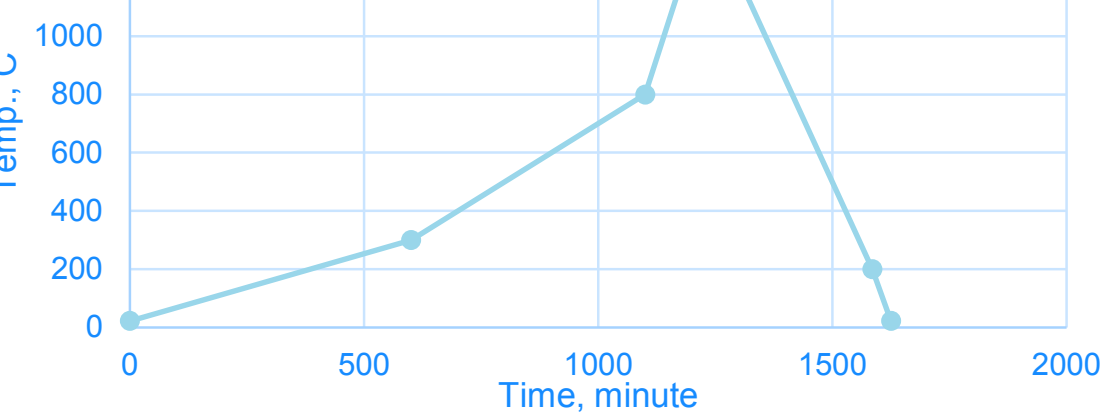

Element * Wt\% | Wt\% Sigma

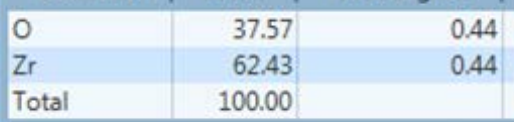

Total 


\section{Zirconia Nanofiber- Yttrium doped}

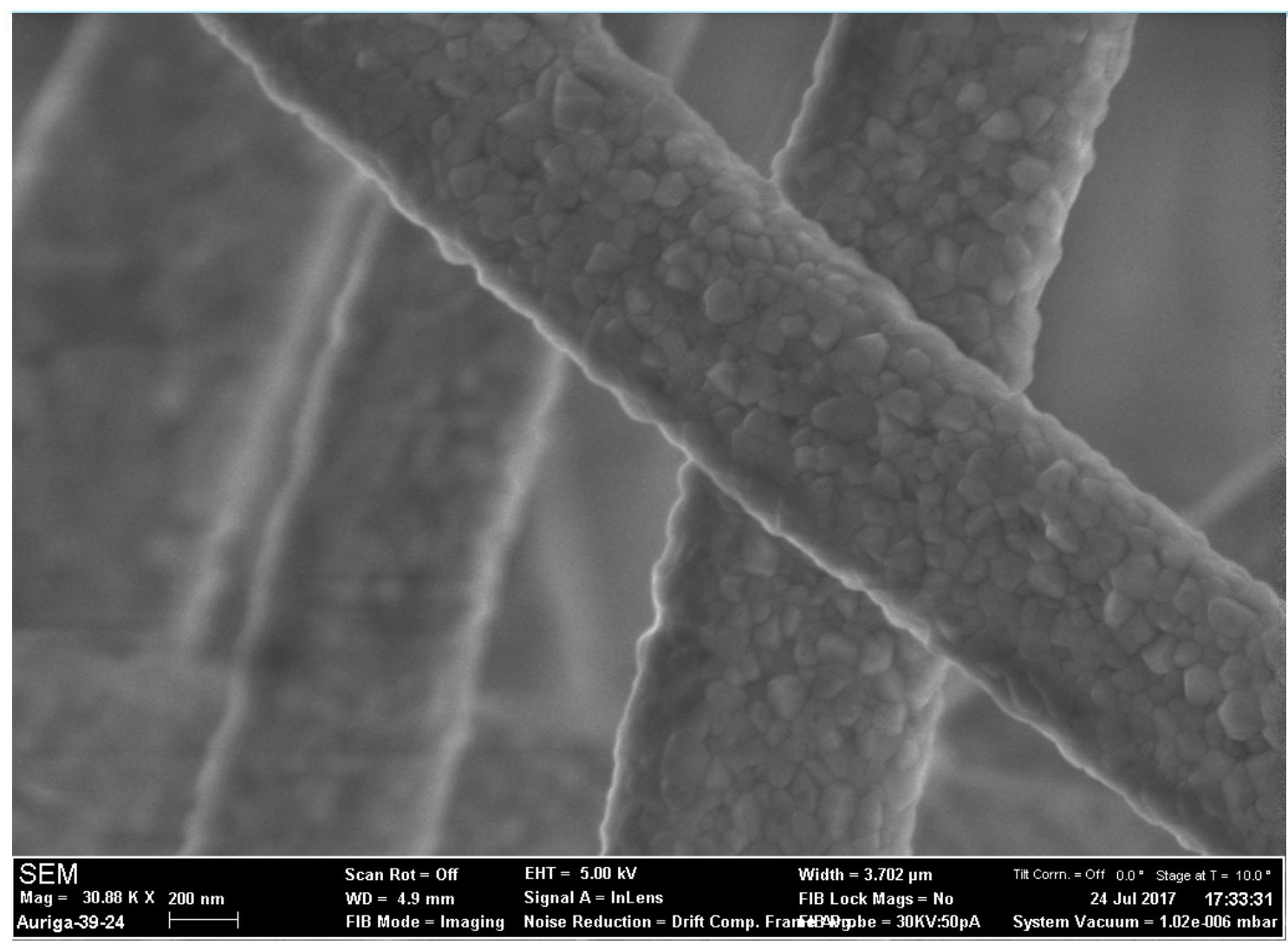

Improve thermal shock resistance by Yttrium doping

Improve radiation resistance

- More grain boundaries blocks dislocation, defect movements, defect recombination*.

- YSZ strong resistance to amorphization 


\section{CNT-Ceramics Nanofiber Composites}

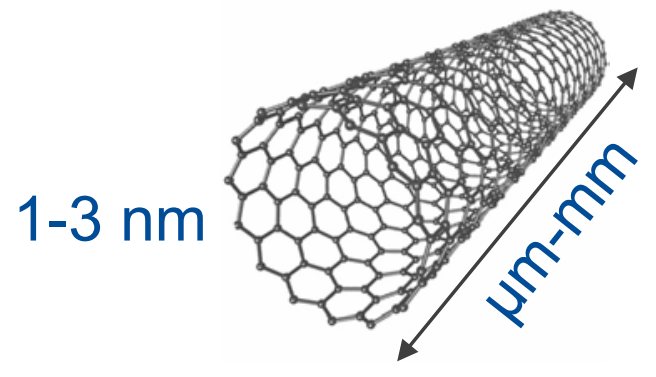

SWCNT
Excellent mechanical properties

E : 1 5 Tpa

Tensile strength : 15-50 Gpa

Elongation \% : 16\%

High thermal conductivity (axial), insulator lateral

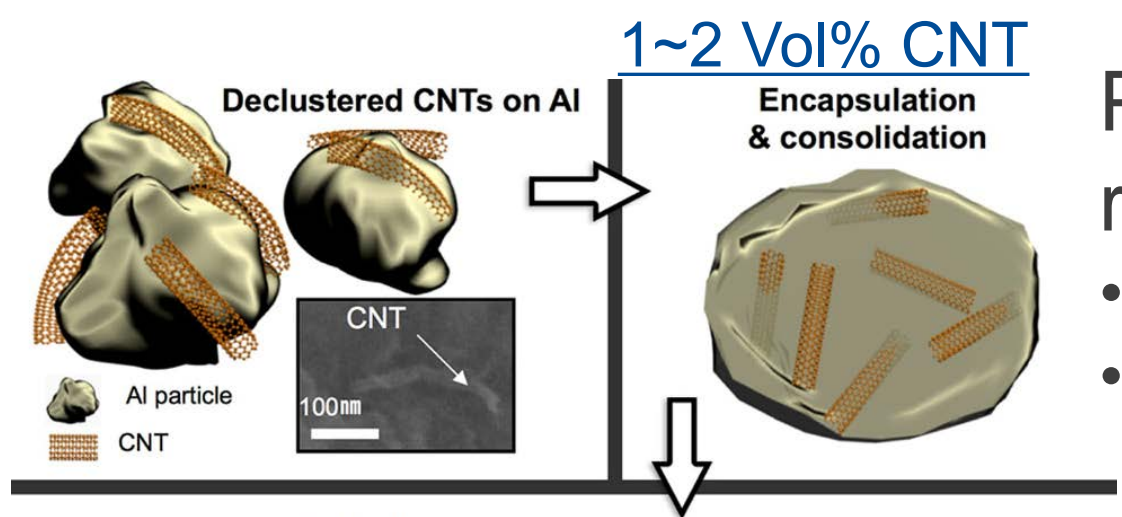

Protects Aluminum metal against radiation damage 70 DPA

- 1-D transport network for He to escape - Reduce embrittlement by 5-10 times.

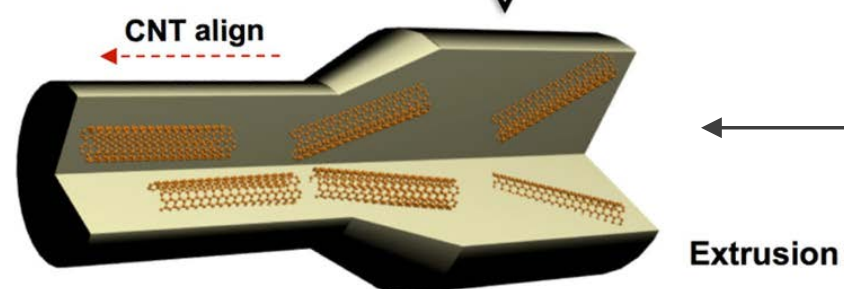

MIT-research*

Bulk not nanofiber!! 


\section{CNT-Zirconia/Alumina Proposed Nanofiber}
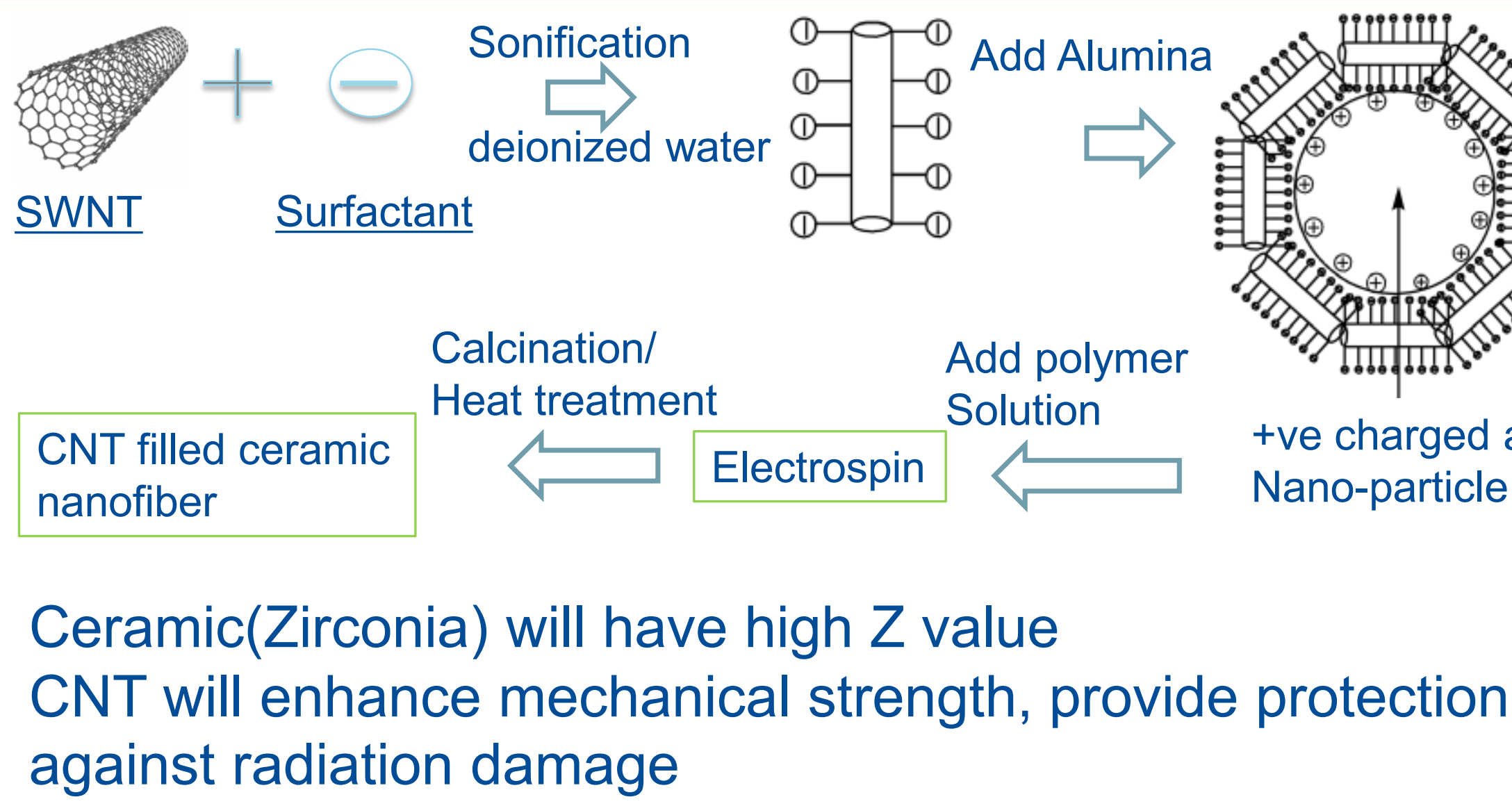
Nano-mechanical mapping Atomic Force Microscopy

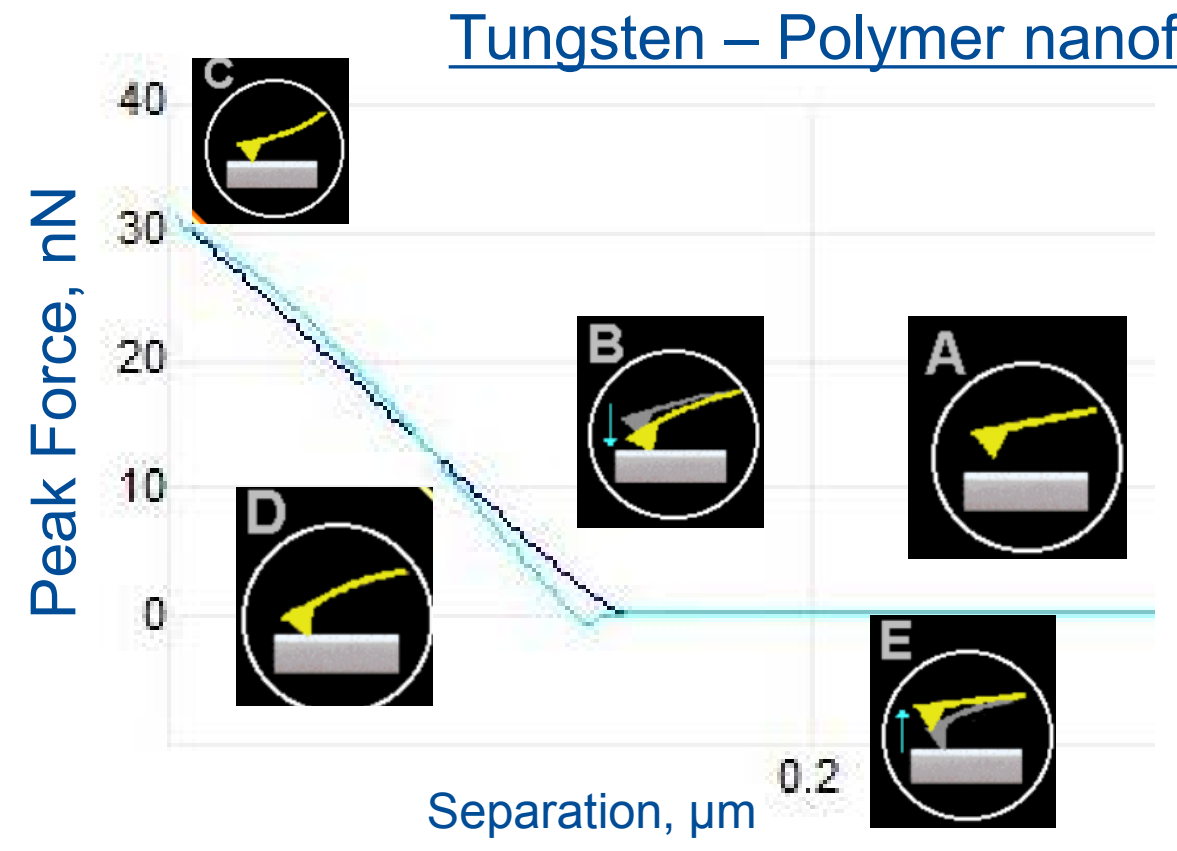

Tip Deflection in contact mode

$F-F_{a d h}=\frac{4}{3} E^{*} V R\left(d-d_{0}\right)^{3}$

Elastic Modulus map

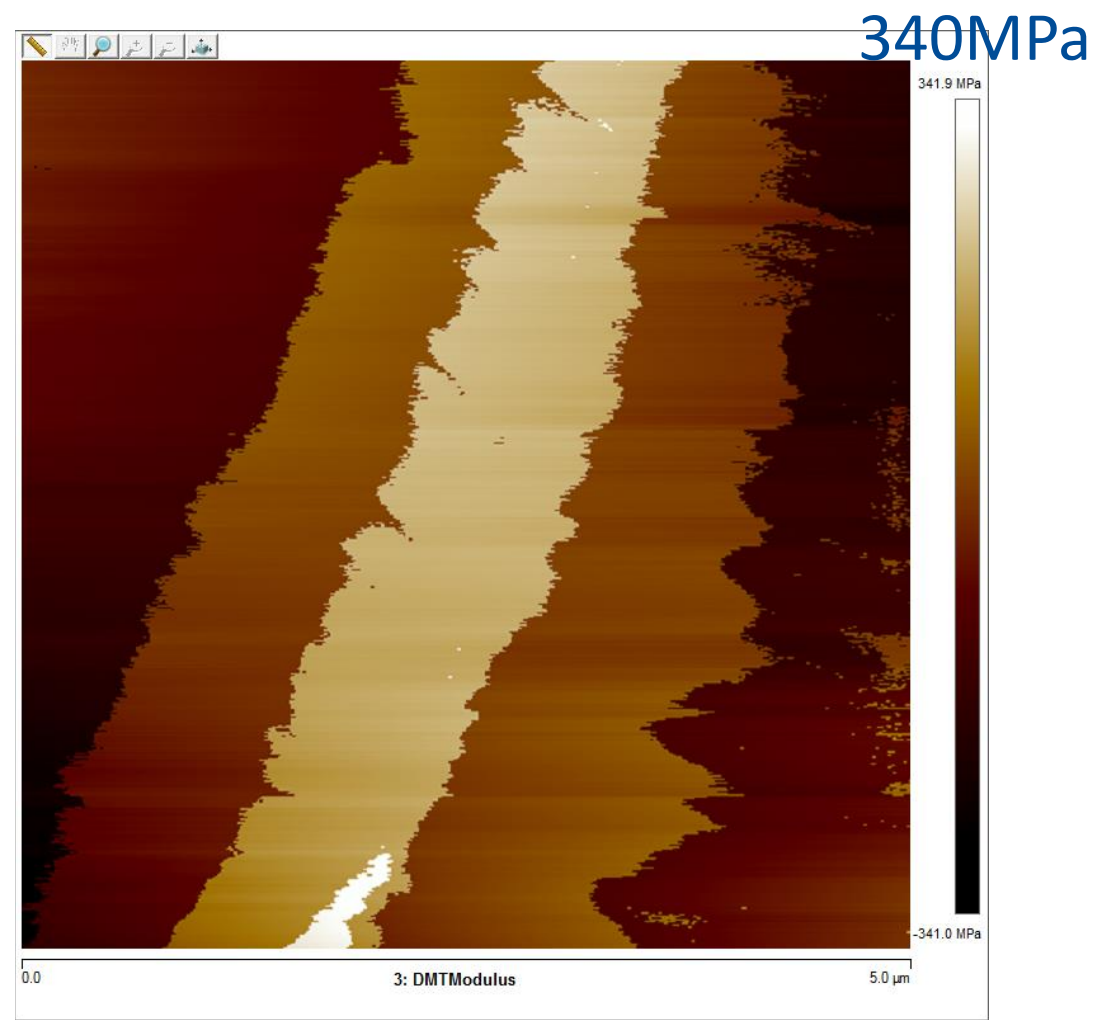

Nanofibers fixed to substrate using double sided tape Soft substrate compared to nanofiber 
Nano-mechanical mapping Atomic Force Microscopy

Elastic Modulus map
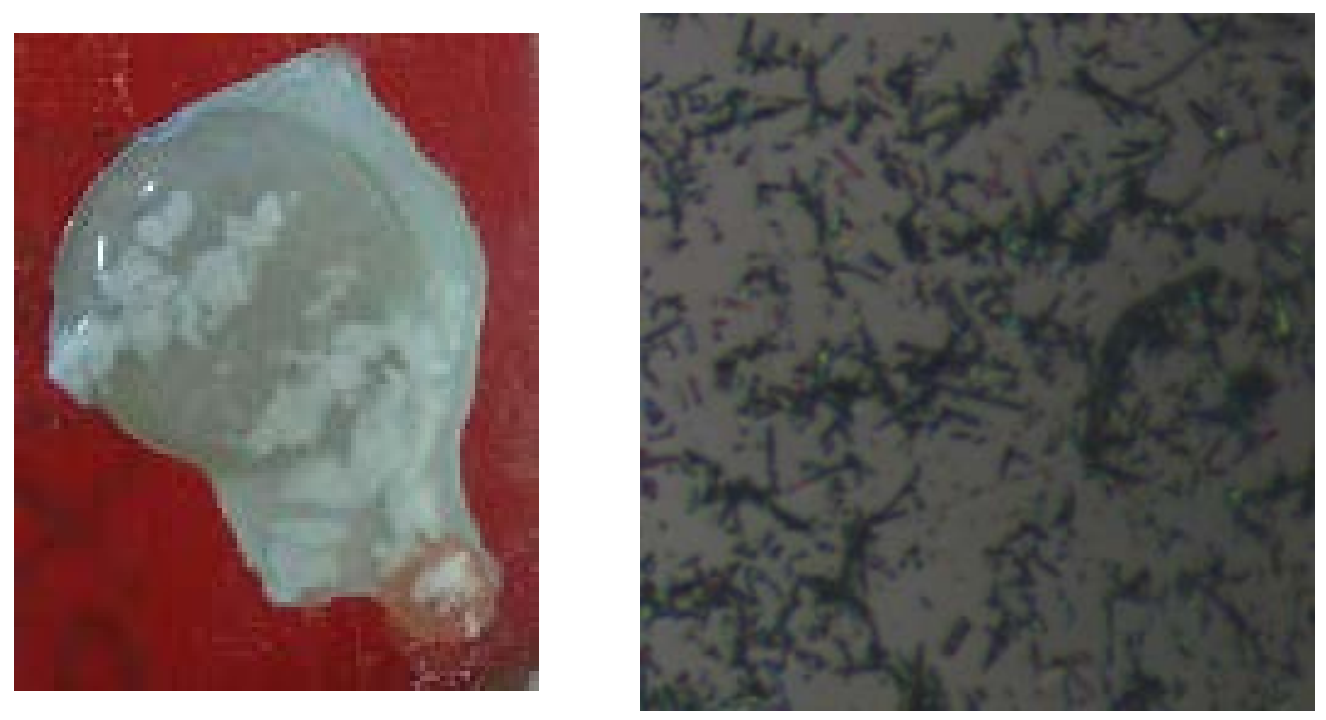

Nanofiber solution casted on harder smooth mica substrate

Average Elastic modulus 100GPa

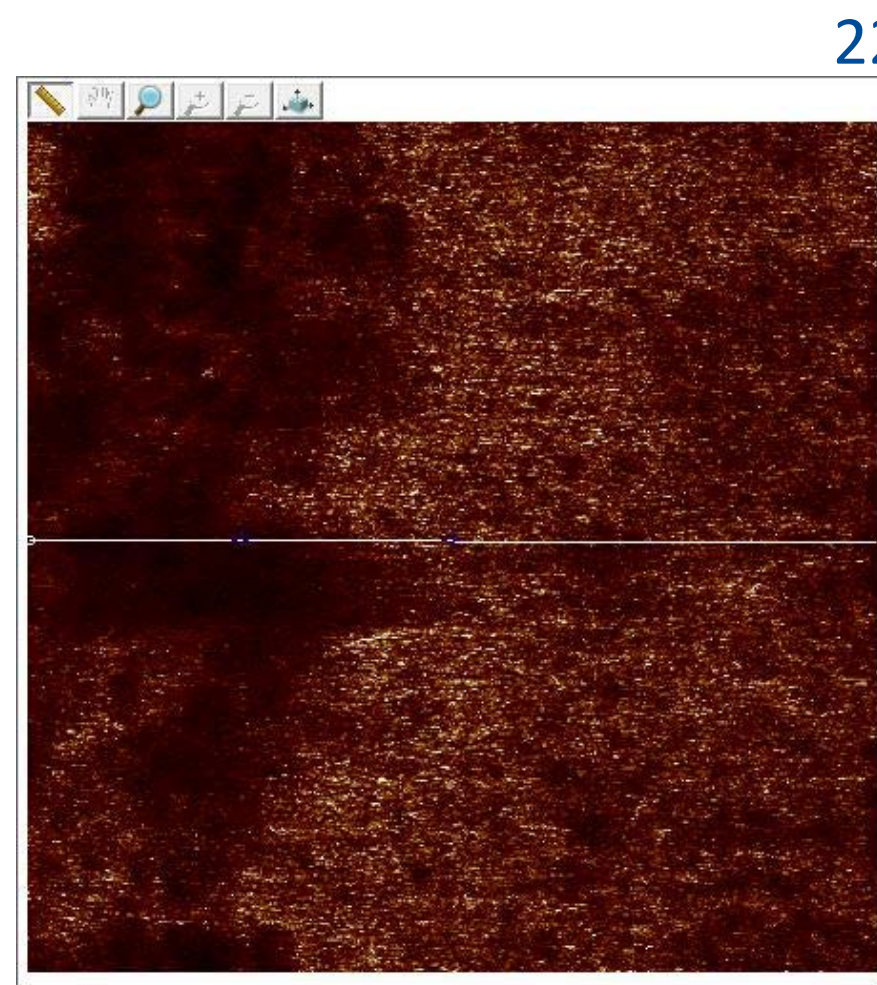

$225 \mathrm{GPa}$

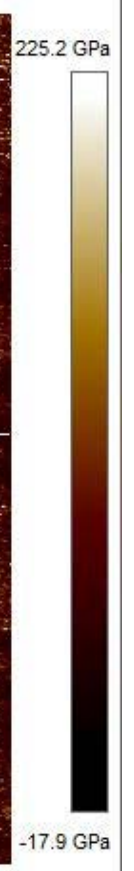

200G Pla

150GPB

100GPia

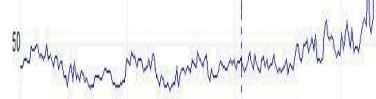

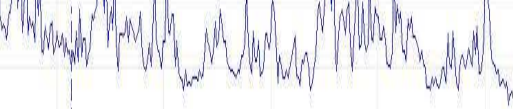

$\mathrm{OP}$

n

16 葉 Fermilab 


\section{Mechanical characterization}

Fracture strength single nanofiber

1. 3 point Bending test on TEM Grid

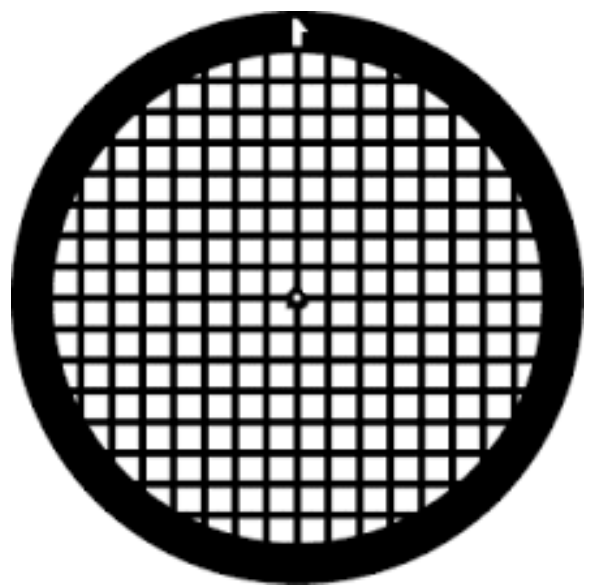

Set up

- Solution cast

- Fix to TEM grid using lon beam (Pt tape)

- Press using diamond AFM tip

2. Nano-indentation using AFM tip
Macro testing electrospun nanofiber mat

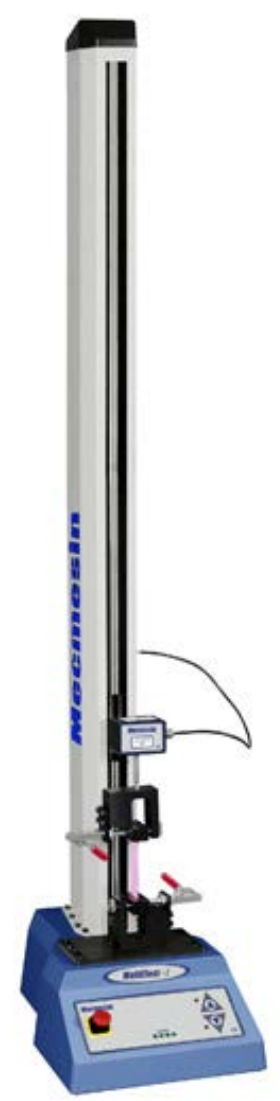




\section{Propose target shape}
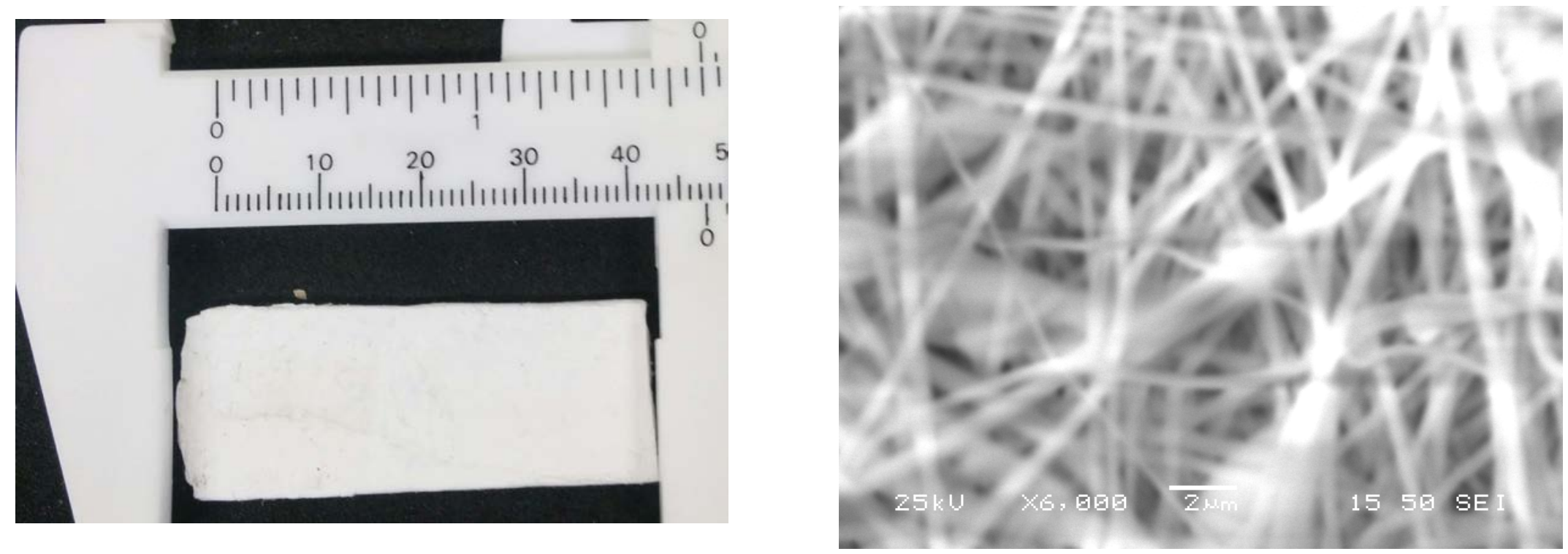

Alumina nano-fiber block

No major modification to current target holder

Customizable

Cheaper and scalable 


\section{Physical Properties Characterization (In progress)}

- Raman spectroscopy:

- disorderness, bond information

- Electron energy loss spectrometry (EELS):

- sp2/sp3 ratio, atomic composition (low Z)

- Wide Angle X-ray Diffraction (WAXD):

- lattice parameters, orientation, isotropy.

- Thermal analysis:

- DSC and DMA :melting and glass transition temperature 


\section{Summary and Future Work}

- Installation of a low cost electrospinning set up completed.

- Success in fabricating metallic and ceramic nanofiber.

- Physical properties of single nanofiber evaluation in progress.

Future work

- Expose nanofiber mat to HiRadMat test

- Single fiber radiation damage study

- Improve ductility of ceramic nanofiber

- Fabricate ceramics-CNT composite.

- Heat treatment profile

- Physical properties before and after radiation.

- Damage modeling 


\section{Thank You for Your Attention!!}




\section{Parameters}

- Molecular weight of polymer

- Solution properties (Viscosity, conductivity, surface tension)

- Concentration, electrical potential, flow rate

- Distance between needle and collector

- Needle gage

- Ambient temperature, humidity, air flow 


\section{Problems}

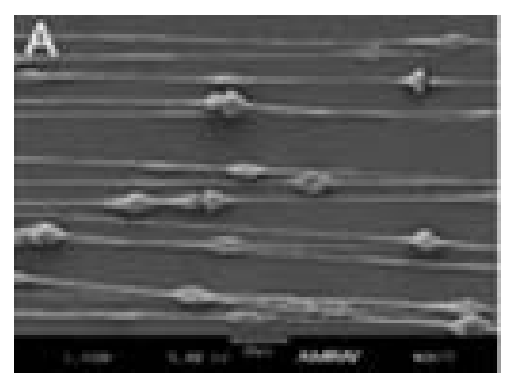

Beading

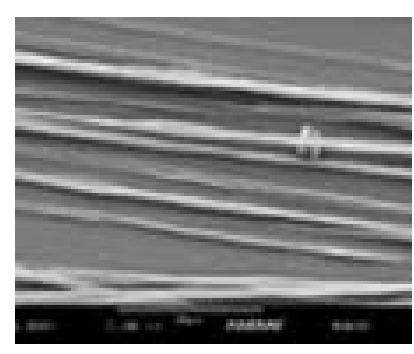

Ribbons

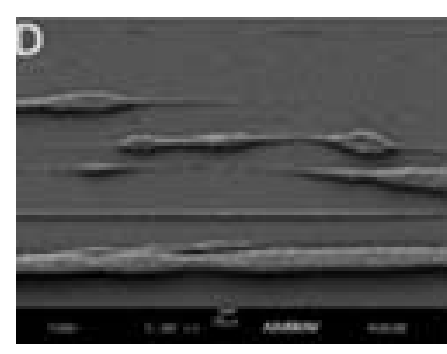

Porous globe

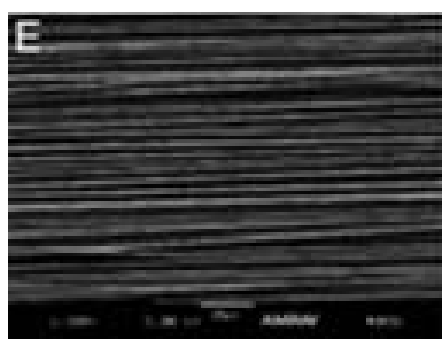

Cylindrical fiber

Troubleshoot

- Increase flow rate

- Increase polymer concentration or solvent with high evaporation

- Salt additives (Surfactant)

- Adjust distance between needle and collector

- Porosity $\rightarrow$ evaporative characteristic of solvent 


\section{Mass Production-Needleless (In progress)}

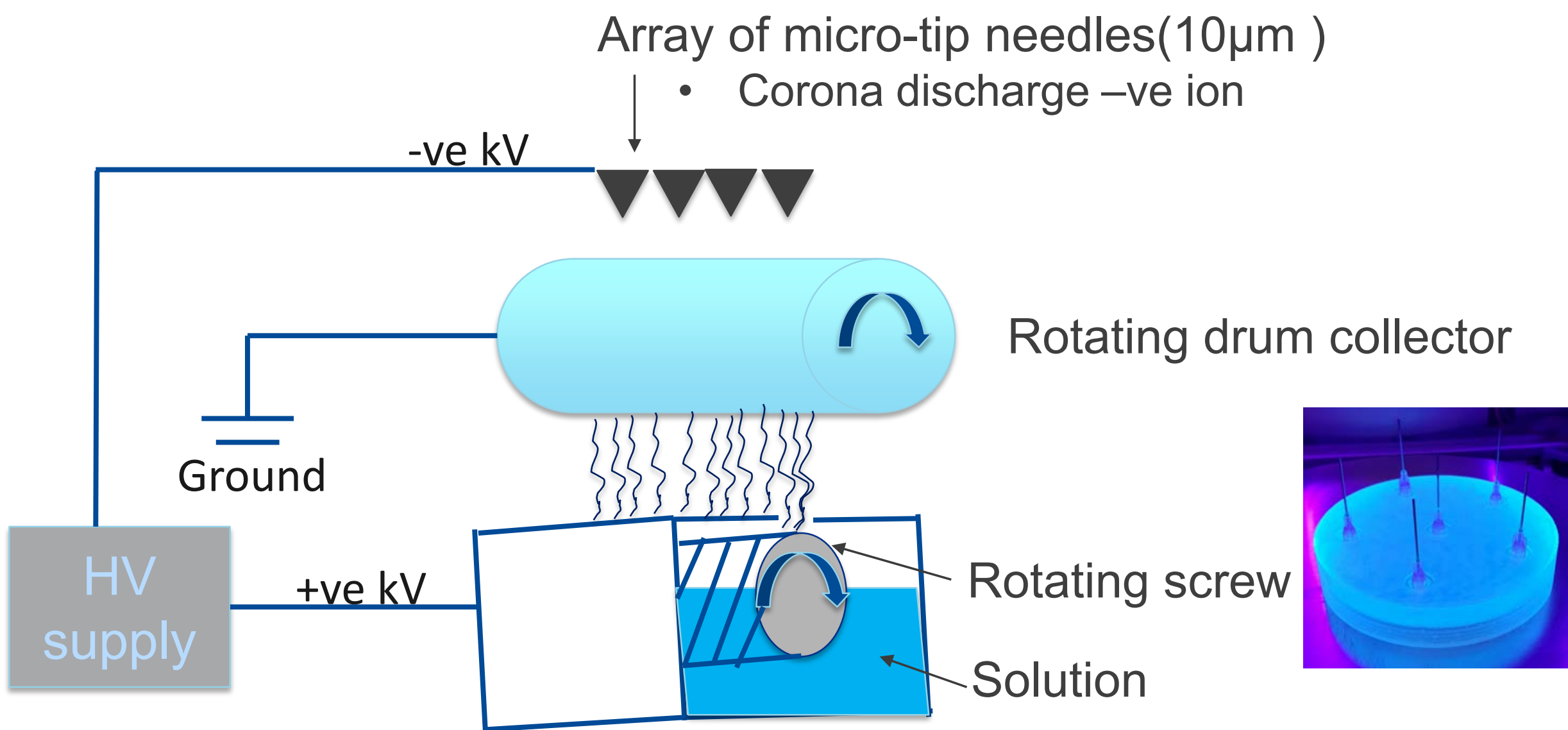

- Charged nanofiber prevents thicker mat formation

- Opposite charged ion neutralize nanofiber and promote thicker mat 